\title{
GEOTHERMAL DATA FROM TEST WELLS DRILLED IN GRASS VALLEY AND BUFFALO VALLEY, NEVADA* +
}

J. H. Sass, F. H. Olmsted, M. L. Sorey, A. H. Lachenbruch, R. J. Munroe, and S. P. Galanis, Jr.

U. S. Geological Survey

Menlo Park, California 94025

and

H. A. Wollenberg

Lawrence Berkeley Laboratory

Univers ity of California

Berkeley, California 94720

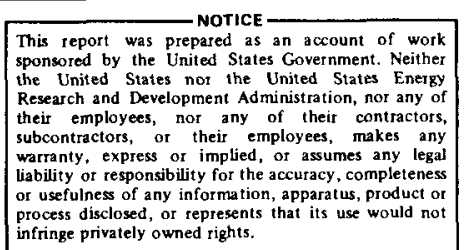

July 1976

*Work partially supported by U. S. Energy Research and Development Administration.

${ }^{\dagger}$ Also released as U. S. Geological Survey Open File Report 76-85. 
Table of Contents

Introduction

Acknowledgments

Leach Hot Springs (Grass Valley) 4

Buffalo Valley Hot Springs

Summary and Conclusions _... 8

References - 9 


\section{DISCLAIMER}

This report was prepared as an account of work sponsored by an agency of the United States Government. Neither the United States Government nor any agency Thereof, nor any of their employees, makes any warranty, express or implied, or assumes any legal liability or responsibility for the accuracy, completeness, or usefulness of any information, apparatus, product, or process disclosed, or represents that its use would not infringe privately owned rights. Reference herein to any specific commercial product, process, or service by trade name, trademark, manufacturer, or otherwise does not necessarily constitute or imply its endorsement, recommendation, or favoring by the United States Government or any agency thereof. The views and opinions of authors expressed herein do not necessarily state or reflect those of the United States Government or any agency thereof. 


\section{DISCLAIMER}

Portions of this document may be illegible in electronic image products. Images are produced from the best available original document. 


\section{INTRODUCTION}

A systematic evaluation of several geothermal prospects in northern Nevada has been carried out by the Lawrence Berkeley Laboratory (LBL) of the University of California during the past two years (Beyer and Morrison, 1976; Bowman and others, 1976; Corwin, 1975; Wollenberg and others, 1975; Majer and others, 1975). Concurrently the U.S. Geological Survey (USGS) has been making a hydrogeologic appraisal and a regional heatflow study of these and other areas in northern and central Nevada (Hose and Taylor, 1974; 01msted and others, 1975; Sass and others, 1975; White and Williams, 1975). This report presents heat-flow data obtained during a cooperative LBL-USGS study of the heat flow and hydrology of two of these prospects, Leach Hot Springs and Buffalo Valley Hot Springs. Figure 1 shows the areas studied in relation to the major towns in the region (Winnemucca and Battle Mountain) together with previously published heat-flow values from this portion of the Battle Mountain heat-flow high (Sass and others, 1971; Diment and others, 1975).

Four types of measurement are considered in this report:

1) Regional background heat flows: For the area shown in Figure 1, all of these data were obtained from crystalline rocks in holes drilled for mineral exploration (Roy and others, 1968; Sass and others, 1971).

2) Shallow hydrologic test wells: These have been drilled in the sedimentary formations surrounding several of the hot springs of northern and central Nevada (01msted and others, 1975) in patterns similar to that of sites $\mathrm{H}-1$ through $\mathrm{H}-15$ (Figure 2). The wells typically are in 
the range of from 20 to $50 \mathrm{~m}$ in depth within a radius of $u 3 \mathrm{~km}$ of the observed surface hydrothermal phenomenon.

3) Heat-flow test wells: Three have been drilled in each of the study areas (Figure 1). These wells are typically in the depth range 100 to $200 \mathrm{~m}$, are cored at two or more intervals of from 1 to $3 \mathrm{~m}$, and have a $32 \mathrm{~mm}$ I.D. access pipe (capped at the bottom) grouted in place to total depth to facilitate temperature measurements.

4) Heat flow - hydrologic test wells: This type of construction has been designated as $Q-H$ in Figure 2 and other tables and figures in this report. They differ from type 3 (above) only in that a parallel pipe with a well screen on the bottom is also emplaced. The annulus of the lowermost $10 \mathrm{~m}$ or so of the wells is packed off with gravel to allow access of formation water. Above the screen, the annulus between the parallel casings and borehole wall is sealed off with cement grout.

The results of the study are shown as a series of graphs and tables along with our preliminary interpretation of the results. Figures 2 through 10 and Tables 1 through 10 present the results from Grass Valley (Leach Hot Springs). Thermal data from the Buffalo Valley Hot Springs are given in Figures 11 through 15 and Tables 11 and 12.

Temperatures and thermal conductivities were determined by the methods described by Sass and others (1971). Most thermal conductivities were measured using the needle probe (Von Herzen and Maxwe11, 1959), but for competent rocks, steady-state measurements using cylindrical disks 
and the divided bar (see e.g., Birch, 1950) were made. The divided-bar measurements (Tables 6,7, and 12) are those which include estimates of density and porosity $(\rho, \phi)$.

The following symbols and units are used:

$\mathrm{K}$, thermal conductivity $\mathrm{mcal} / \mathrm{cm} \mathrm{sec}{ }^{\circ} \mathrm{C}$

$<K>$, harmonic mean thermal conductivity

$N$, number of thermal conductivity measurements

$\mathrm{q}$, heat flow $(\mathrm{HFU})=\mu \mathrm{cal} / \mathrm{cm}^{2} \mathrm{sec}$

$\rho$, density $\mathrm{g} / \mathrm{cm}^{3}$

$\phi$, porosity (\% voids)

Acknowledgments. Drilling was done by a crew from the U.S. Bureau of Reclamation under the supervision of Ted Darrow. Shirley Crossland and Marcello Lippman helped monitor drilling operations for Grass Valley. We thank Tom Moses and Glen Blevens for technical assistance in well completion. Geophysical logs other than temperature logs were made by Richard McCullough, and needle-probe determinations of thermal conductivity by Eugene Smith. 


\section{LEACH HOT SPRINGS (GRASS VALLEY)}

The major effort to date has been in the vicinity of Leach Hot Springs (Figure 2). Table 1 presents a summary of the heat-flow data obtained from the seven relatively deep wells drilled as part of this study. Table 2 summarizes the results from shallow hydrologic wells (sites $\mathrm{H}-1$ through $\mathrm{H}-15$, Figure 2); it is an updating of thermal data from the hydrogeologic appraisal first presented by $01 \mathrm{msted}$ and others (1975). The measurements at different times in each test well indicate small changes in temperature at depths of 15 and 30 meters and also small differences in temperature gradient. Because these changes occur at depths beneath the annual conductive temperature wave, they must be attributed to some combination of:

1) Longer period conductive variations,

2) Heat transfer by moving water, and

3) Instrumental error. The relative magnitude of each source of variation is not known. The depth intervals for which the temperature gradients are tabulated are those in which the gradients are nearly linear and presumed to be chiefly conductive and steady.

Tables 3 through 9 contain details of lithology and individual thermal conductivity measurements for the various heat-flow test holes. Table 10 shows the stratigraphy based on examination of drill cuttings. Figure 3 is a graph showing temperature profiles from all seven of the heat flow (Q) and heat flow - hydrologic wells $(Q-H)$ drilled between April and June 1975. This figure and the heat-flow determinations 
(Table 1) demonstrate a large variation in conductive heat flows over a relatively small area. Temperature profiles for individual wells obtained in June and October 1975 (Figures 4 through 10) show that the thermal regime in the upper $200 \mathrm{~m}$ is primarily conductive with the annual wave being attenuated between about 12 and $15 \mathrm{~m}$ below the surface.

The large range of heat flows ( 1.4 to $9 \mathrm{HFU}$ ) suggests that a hydrothermal convective system influences the temperatures at depths greater than a few hundred meters below the surface. Combining gradients in the shallow holes near Leach Hot Springs (Table 2) with the relatively high thermal conductivities determined from $\mathrm{QH}-1$ results in conductive heat flows ranging from 1.4 to greater than $60 \mathrm{HFU}$. The gradients further indicate a general pattern of decreasing heat flow out to a radial distance of $2 \mathrm{~km}$ from the springs (see also $01 \mathrm{~ms}$ ted and others, 1975). This reinforces the presumption of a relatively local hydrothermal upflow enclosed by impermeable sedimentary rocks surrounding Leach Hot Springs. The high value at site Q-3 (4.87 HFU) confirms the high heat flows reported earlier by Sass and others (1971) from crystalline rocks at the Big Mike Mine, south of Panther Canyon. This heat flow, together with the high value at site $\mathrm{QH}-3$ (5.11 HFU), flanked by considerably lower heat flows at $\mathrm{QH}-4$ and $\mathrm{Q}-2$, indicate the presence of complex upward and downward flow at depth in Grass Valley, in addition to the flow beneath the orifice at Leach Hot Springs. 


\section{BUFFALO VALLEY HOT SPRINGS}

Three heat-flow holes were drilled near the Buffalo Valley Hot Springs (Figure 11). Hole \#1 was drilled approximately $500 \mathrm{~m}$ east of the easternmost pool of Buffalo Valley Hot Springs, in an area known to be anomalous by previous shallow temperature measurements (01msted and others, 1975). Hole \#2 was drilled $\sim 2 \mathrm{~km}$ west of the hot springs thermal anomaly, to observe "background" heat flow in valley alluvium away from the springs. Hole \#3 was drilled on a low mound in the northwestern portion of the valley playa, on the northern projection of a mid-valley fault zone, considered possibly thermally active (Wollenberg and others, 1975). Temperature profiles from all three holes are shown in Figure 12 and individual profiles obtained on two different dates are plotted in Figures 13 through 15. Unfortunately, the casing could not be lowered below about $37 \mathrm{~m}$ in hole 1 so that the only available temperature $\log$ is one obtained on 10-12-74, a few hours after completion of drilling (Figures 12 and 13). There appears to be a hydrologic effect between depths of about 30 and $40 \mathrm{~m}$ in hole 3 (Figure 15). Apart from this, however, temperature gradients reflect a conductive regime. This, in turn, suggests that relatively impermeable rocks enclose or "cap" the hydrothermal system. Not shown in Figure 11 are the 9 shallow test wells (of the "H" type, Figure 2) drilled near the springs and previously reported by $01 m s t e d$ and others, 1975. From observations in these wells, $01 \mathrm{msted}$ and others (1975) estimated that within a radius of 1 or $2 \mathrm{~km}$ 
heat flows increase sharply to $>50 \mathrm{HFU}$ near the springs. The high heat flow from $B V-1$ about $1 / 2 \mathrm{~km}$ from the springs and the normal value from BV-2 (Figure 11, Table 11) about $12 \mathrm{~km}$ west of the springs generally confirm this view. However, the value of $6.5 \mathrm{HFU}$ at BV-1 is less by a factor of 3 or more than the heat flow estimated for the same region from the shallower wells. 


\section{SUMMARY AND CONCLUSIONS}

Subsurface thermal observations indicate that Leach Hot Springs in Grass Valley, and Buffalo Hot Springs in Buffalo Valley, overlie localized upflows of hot water enclosed by relatively impermeable sediments. Upward and downward flows occur elsewhere in Grass Valley, probably indicating a complex system of hydrothermal circulation. The thermal data are inadequate to determine whether a comparable condition exists in Buffalo Valley.

Deeper test drilling is being planned for both the Leach and Buffalo Hot Spring areas to find the depth to which the conductive regime extends and to provide further sub-surface data for an evaluation of the geothermal energy potential of the prospects. 


\section{References}

Beyer, J.H., and Morrison, H. F., Electrical exploration of geothermal systems in Basin and Range valleys of Nevada: Proc., Second U.N. Symposium on the Development and Use of Geothermal Resources, in press, 1976.

Birch, F., 1950, Flow of heat in the Front Range, Colorado: Geol. Soc. America Bu11., v. 61, p. 567-630.

Bowman, H. R., Herbert, A. J., Wollenberg, H. A., and Asaro, F., Trace, minor and major elements in geothermal waters and associated rock formations (north-central Nevada): Proc., Second U.N. Symposium on the Development and Use of Geothermal Resources, in press, 1976.

Corwin, R. F., 1975, Use of the self-potential method for geothermal exploration: Lawrence Berkeley Laboratory Rept. LBL 3235.

Diment, W. H., Urban, T. C., Sass, J. H., Marshal1, B. V., Munroe, R. J., and Lachenbruch, A. H., 1975, Temperatures and heat contents based on conductive transport of heat, in White, D. E., and Williams, D. L., eds., Assessment of geothermal resources of the United States-1975: U.S. Geol. Survey Circ. 726, p. 84-103.

Hose, R. K., and Taylor, B. E., 1974, Geothermal systems of northern Nevada: U.S. Geol. Survey Open-File Rept. 74-271, 27 pp.

Majer, E., Liaw, A., and McEvilly, T. V., 1975, Seismological investigations near Leach Hot Springs, Nevada (abs.): EOS (Anı. Geophys Union Trans.), v. 56, no. 12, p. 
01msted, F. H., Glancy, P. A., Harrill, J. R., Rush, F. E., and VanDenburgh, A. S., 1975, Preliminary hydrogeologic appraisal of selected hydrothermal systems in northern and central Nevada: U.S. Geol. Survey Open-File Rept. 75-56, 267 pp.

Roy, R. F., Decker, E. R., Blackwe11, D. D., and Birch, Francis, 1968, Heat flow in the United States: Jour. Geophys. Research, v. 73, p. 52075221.

Sass, J. H., Lachenbruch, A. H., Munroe, R. J., Greene, G. W., and Moses, T. H., Jr., 1971, Heat flow in the western United States: Jour. Geophys. Research, v. 76, p. 6376-6413.

Sass, J. H., Lachenbruch, A. H., Diment, W. H., and Urban, T. C., 1975, Heat-flow patterns and geothermal resource potential of the western United States (abs.): Second U.N. Symposium on the Development and Use of Geothermal Resources, San Francisco, California, May 1975, abstract no. III -80 .

Von Herzen, R. P., and Maxwel1, A. E., 1959, The measurement of thermal conductivity of deep-sea sediments by a needle probe method: Jour. Geophys. Research, v. 64, p. 1557-1563.

White, D. E., and Williams, D. L., eds., 1975, Assessment of geothermal resources of the United States--1975: U.S. Geol. Survey Circ. 726, $155 \mathrm{pp}$.

Woll enberg, H. A., Asaro, F., Bowman, H., McEvilly, T., Morrison, F., and Witherspoon, P., 1975, Geothermal energy resource assessment: Lawrence Berkeley Laboratory Rept. UC1D 3762. 
TABLE 1. Summary of temperature gradients, conductivities, and heat flows for holes near Leach Hot Springs

\begin{tabular}{lcccc}
\hline Hole & $\begin{array}{c}\text { Gradient } \\
{ }^{\circ} \mathrm{C} / \mathrm{km}\end{array}$ & i $*$ & $\begin{array}{c}\mathrm{K}^{* *} \\
\mathrm{mca} / / \mathrm{cm} \text { sec }\end{array}{ }^{\circ} \mathrm{C}$ & $\begin{array}{c}\mathrm{q} \\
\mathrm{HFU}\end{array}$ \\
\hline Q1 & 65.5 & 12 & 3.42 & 2.24 \\
Q2 & 55.5 & 10 & 3.68 & 2.04 \\
$\mathrm{Q3}$ & 120 & 16 & 4.06 & 4.87 \\
$\mathrm{QH}-1$ & 224 & 12 & 4.03 & 9.03 \\
$\mathrm{QH}-2$ & 52 & 17 & 2.88 & 1.50 \\
$\mathrm{QH}-3$ & 118 & 9 & 4.33 & 5.11 \\
$\mathrm{QH}-4$ & 42 & 11 & 3.25 & 1.36 \\
\hline
\end{tabular}

* Number of conductivity determinations. ** Harmonic mean thermal conductivity (see Tables 3 through 9 for individual determinations). 
TABLE 2. Shal low temperatures and temperature gradients for U.S. Geological Survey shallow test holes, Grass Valley, Nevada

\begin{tabular}{|c|c|c|c|c|c|c|c|c|}
\hline \multirow[b]{2}{*}{$\begin{array}{l}\text { Test } \\
\text { nole* }\end{array}$} & \multicolumn{3}{|c|}{$\begin{array}{l}\text { Date of } \\
\text { measurement }\end{array}$} & \multirow{2}{*}{$\begin{array}{l}\text { Depth to } \\
\text { saturated } \\
\text { zone } \\
(m)\end{array}$} & \multicolumn{2}{|c|}{$\begin{array}{l}\text { Temperature } \\
\left({ }^{\circ} \mathrm{C}\right)\end{array}$} & \multirow{2}{*}{$\begin{array}{l}\text { Depth interval } \\
\text { measured } \\
(\mathrm{m})\end{array}$} & \multirow{2}{*}{$\begin{array}{l}\text { Temperature } \\
\text { gradient } \\
\left({ }^{\circ} \mathrm{C} / \mathrm{km}\right)\end{array}$} \\
\hline & $\stackrel{\infty}{\stackrel{\infty}{\infty}}$ & 읕 & ते & & $15 \mathrm{~m}$ & $30 \mathrm{~m}$ & & \\
\hline \multirow{4}{*}{$\begin{array}{l}H-1 \\
P, S c\end{array}$} & 73 & 06 & 14 & & 13.82 & 14.80 & $12.98-44.84$ & 64 \\
\hline & 73 & 12 & 14 & $12.6^{\mathrm{N}}$ & 13.42 & 14.38 & $13.59-44.68$ & 70 \\
\hline & 74 & 08 & 07 & & 13.70 & 14.46 & $13.85-44.85$ & 63 \\
\hline & 75 & 06 & 21 & & 13.40 & 14.31. & $17.00-45.00$ & 69 \\
\hline \multirow{9}{*}{$\begin{array}{l}H-2 \\
P, C\end{array}$} & 73 & 06 & 15 & & 15.38 & 15.73 & $15.12-50.14$ & 20 \\
\hline & 73 & 12 & 14 & $>50$ & 14.26 & 14.98 & $13.90-40.72$ & 50 \\
\hline & & & & & & & $40.72-50.05$ & 32 \\
\hline & 74 & 08 & 06 & & 14.15 & 14.97 & $15.54-40.54$ & 49 \\
\hline & & & & & & & $40.54-49.54$ & 36 \\
\hline & 75 & 04 & 24 & & 14.15 & 14.85 & $14.00-40.00$ & 49 \\
\hline & & & & & & & $40.00-50.03$ & 37 \\
\hline & 74 & 06 & 23 & & 14.06 & 14.87 & $14.00-30.00$ & 52 \\
\hline & & & & & & & $34.00-42.00$ & 44 \\
\hline \multirow{8}{*}{$\begin{array}{l}H-3 \\
P, C\end{array}$} & 73 & 06 & 15 & & 24.95 & 32.92 & $11.31-23.50$ & 550 \\
\hline & & & & & & & $23.50-49.87$ & 490 \\
\hline & 73 & 12 & 14 & $22-26^{N}$ & 25.07 & 33.35 & $12.53-23.50$ & 610 \\
\hline & & & & & & & $23.50-49.96$ & 480 \\
\hline & 75 & 04 & 24 & & 25.04 & 33.45 & $14.00-23.00$ & 610 \\
\hline & & & & & & & $23.00-50.09$ & 480 \\
\hline & 75 & 06 & 22 & & 25.00 & 33.35 & $14.00-22.00$ & 650 \\
\hline & & & & & & & $22.00-46.00$ & 490 \\
\hline
\end{tabular}


TABLE 2. Shallow temperatures and temperature gradients for U.S. Geological Survey shallow test holes, Grass Valley, Nevada (continued)

\begin{tabular}{|c|c|c|c|c|c|c|c|c|}
\hline \multirow[b]{2}{*}{$\begin{array}{l}\text { Test } \\
\text { hole* }\end{array}$} & \multicolumn{3}{|c|}{$\begin{array}{c}\text { Date of } \\
\text { measurement }\end{array}$} & \multirow{2}{*}{$\begin{array}{l}\text { Depth to } \\
\text { saturated } \\
\text { zone } \\
\text { (m) }\end{array}$} & \multicolumn{2}{|c|}{$\begin{array}{l}\text { Temperature } \\
\left({ }^{\circ} \mathrm{C}\right)\end{array}$} & \multirow{2}{*}{$\begin{array}{l}\text { Depth interval } \\
\text { measured } \\
(\mathrm{m})\end{array}$} & \multirow{2}{*}{$\begin{array}{l}\text { Temperature } \\
\text { gradient } \\
\left({ }^{\circ} \mathrm{C} / \mathrm{km}\right)\end{array}$} \\
\hline & $\stackrel{\pi}{\infty}$ & $\stackrel{\circ}{E}$ & ते & & $15 \mathrm{~m}$ & $30 \mathrm{~m}$ & & \\
\hline$H-4$ & 73 & 06 & 19 & & 20.81 & 25.48 & $14.97-23.50$ & 330 \\
\hline \multirow[t]{10}{*}{$P, C$} & & & & & & & $23.50-47.88$ & 260 \\
\hline & 73 & 12 & 14 & $23.6^{N}$ & 20.61 & 25.36 & $13.75-24.72$ & 380 \\
\hline & & & & & & & $24.72-38.25$ & 210 \\
\hline & & & & & & & $33.25-45.45$ & 270 \\
\hline & 75 & 04 & 24 & & 20.63 & 25.35 & $14.00-18.00$ & 340 \\
\hline & & & & & & & $18.00-24.00$ & 370 \\
\hline & & & & & & & $24.00-44.00$ & 260 \\
\hline & 75 & 06 & 13 & $23.9^{N}$ & 20.75 & 25.35 & $14.00-26.00$ & 350 \\
\hline & & & & & & & $26.00-34.00$ & 200 \\
\hline & & & & & & & $34.00-49.90$ & 260 \\
\hline \multirow{6}{*}{$\begin{array}{l}\mathrm{H}-5 \\
\mathrm{P}, \mathrm{C}\end{array}$} & 73 & 07 & 08 & & 16.02 & $18.5^{\mathrm{e}}$ & $13.44-19.54$ & 180 \\
\hline & & & & & & & $19.54-27.16$ & 160 \\
\hline & 73 & 12 & 14 & $>27$ & 15.63 & $18.1^{\mathrm{e}}$ & $13.44-19.54$ & 180 \\
\hline & & & & & & & $19.54-27.13$ & 150 \\
\hline & 75 & 06 & 14 & & 15.55 & & $14.00-22.00$ & 190 \\
\hline & & & & & & & $22.00-27.13$ & 160 \\
\hline & 73 & 07 & 09 & & $17.9^{\mathrm{e}}$ & 20.45 & $29.90-40.26$ & 180 \\
\hline \multirow{4}{*}{ St, Sc } & 73 & 12 & 14 & $16.7^{\mathrm{N}}$ & $17.7^{\mathrm{e}}$ & 20.18 & $29.29-40.26$ & 180 \\
\hline & 74 & 08 & 07 & & $17.7^{\mathrm{e}}$ & 20.27 & $30.09-40.09$ & 170 \\
\hline & 75 & 04 & 24 & & $17.8^{\mathrm{e}}$ & 20.20 & $30.00-40.00$ & 170 \\
\hline & 75 & 06 & 21 & & & 20.25 & $30.00-41.00$ & 170 \\
\hline
\end{tabular}


14

TABLE 2. Shallow temperatures and temperature gradients

for U.S. Geological Survey shallow test holes, Grass Valley, Nevada (continued)

\begin{tabular}{|c|c|c|c|c|c|c|c|c|}
\hline \multirow[b]{2}{*}{$\begin{array}{l}\text { Test } \\
\text { hole* }\end{array}$} & \multicolumn{3}{|c|}{$\begin{array}{l}\text { Date of } \\
\text { measurement }\end{array}$} & \multirow{2}{*}{$\begin{array}{l}\text { Depth to } \\
\text { saturated } \\
\text { zone } \\
\text { (m) }\end{array}$} & \multicolumn{2}{|c|}{$\begin{array}{l}\text { Teniperature } \\
\left({ }^{\circ} \mathrm{C}\right)\end{array}$} & \multirow{2}{*}{$\begin{array}{l}\text { Depth interval } \\
\text { measured } \\
(\mathrm{m})\end{array}$} & \multirow{2}{*}{$\begin{array}{l}\text { Temperature } \\
\text { gradient } \\
\left({ }^{\circ} \mathrm{C} / \mathrm{km}\right)\end{array}$} \\
\hline & 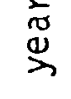 & $\stackrel{O}{E}$ & ते & & $15 \mathrm{~m}$ & $30 \mathrm{~m}$ & & \\
\hline $\mathrm{H}-7$ & 73 & 07 & 09 & & & 17.83 & $27.13-50.90$ & 100 \\
\hline \multirow[t]{3}{*}{$P, S c$} & 73 & 12 & 15 & $26.4^{N}$ & & 17.54 & $33.22-50.29$ & 110 \\
\hline & 74 & 08 & 07 & & & 17.58 & $33.45-48.45$ & 100 \\
\hline & 75 & 06 & 21 & & & 17.63 & $30.00-50.88$ & 110 \\
\hline$H-8$ & 73 & 10 & 31 & & 12.66 & 13.70 & $19.02-44.32$ & 67 \\
\hline \multirow[t]{5}{*}{$P, S C$} & 73 & 11 & 09 & $22.9^{N}$ & & 13.77 & $27.55-44.01$ & 69 \\
\hline & 73 & 12 & 13 & & & 13.86 & $24.51-44.62$ & 68 \\
\hline & 74 & 08 & 06 & & & 13.84 & $26.79-44.79$ & 66 \\
\hline & 74 & 12 & 10 & & & 13.65 & $28.00-45.00$ & 67 \\
\hline & 75 & 06 & 21 & & & 13.68 & $28.00-44.95$ & 68 \\
\hline \multirow{9}{*}{$\begin{array}{l}H-9 \\
S t, S C\end{array}$} & 73 & 11 & 01 & $36.2^{N}$ & & 37.50 & $28.07-35.39$ & 710 \\
\hline & & & & & & & $35.39-42.70$ & 660 \\
\hline & 73 & 11 & 09 & & & 38.62 & $28.07-35.39$ & 680 \\
\hline & & & & & & & $35.39-41.48$ & 610 \\
\hline & 73 & 12 & 15 & & & 38.80 & $28.07-35.39$ & 720 \\
\hline & & & & & & & $35.39-42.09$ & 630 \\
\hline & 74 & 08 & 07 & & & $39.2^{\mathrm{e}}$ & $31.09-36.09$ & 630 \\
\hline & & & & & & & $36.09-42.61$ & 640 \\
\hline & 75 & 06 & 21 & & & $38.7^{e}$ & $35.00-41.00$ & 640 \\
\hline
\end{tabular}


TABLE 2. Shallow temperatures and temperature gradients

for U.S. Geological Survey shallow test holes, Grass Valley, Nevada (continued)

\begin{tabular}{|c|c|c|c|c|c|c|c|c|}
\hline \multirow[b]{2}{*}{$\begin{array}{l}\text { Test } \\
\text { hole* }\end{array}$} & \multicolumn{3}{|c|}{$\begin{array}{c}\text { Date of } \\
\text { measurement }\end{array}$} & \multirow{2}{*}{$\begin{array}{l}\text { Depth to } \\
\text { saturated } \\
\text { zone } \\
\text { (m) }\end{array}$} & \multicolumn{2}{|c|}{$\begin{array}{l}\text { Temperature } \\
\left({ }^{\circ} \mathrm{C}\right)\end{array}$} & \multirow{2}{*}{$\begin{array}{l}\text { Depth interval } \\
\text { measured } \\
(\mathrm{m})\end{array}$} & \multirow{2}{*}{$\begin{array}{l}\text { Temperature } \\
\text { gradient } \\
\left({ }^{\circ} \mathrm{C} / \mathrm{km}\right)\end{array}$} \\
\hline & 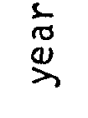 & $\stackrel{\circ}{E}$ & $\overrightarrow{0}$ & & $15 \mathrm{~m}$ & $30 \mathrm{~m}$ & & \\
\hline \multirow{7}{*}{$\begin{array}{l}\mathrm{H}-10 \\
\mathrm{St}, \mathrm{SC}\end{array}$} & 73 & 11 & 09 & $5.8^{c}$ & 82.41 & $108.0^{e}$ & $5.82-9.48$ & 1,400 \\
\hline & & & & & & & $9.48-16.79$ & 1,800 \\
\hline & 73 & 12 & 15 & $5.8^{C}$ & 85.10 & $110.0^{e}$ & $5.82-8.87$ & 1,300 \\
\hline & & & & & & & $8.87-16.79$ & 1,800 \\
\hline & 74 & 08 & 07 & $5.8^{c}$ & 86.55 & $111.7^{\mathrm{e}}$ & $6.39-9.39$ & 1,700 \\
\hline & & & & & & & $9.39-16.76$ & 1,900 \\
\hline & 75 & 06 & 22 & $5.8^{c}$ & 85.50 & & $6.00-16.79$ & 1,700 \\
\hline \multirow{4}{*}{ St, SC. } & 73 & 11 & 09 & $29.5^{\mathrm{N}}$ & & $21.1^{\mathrm{e}}$ & $32.40-44.59$ & 250 \\
\hline & 73 & 12 & 15 & & & 21.22 & $31.18-44.59$ & 240 \\
\hline & 74 & 08 & 07 & & & 21.34 & $35.09-43.65$ & 240 \\
\hline & 75 & 06 & 21 & & & 21.51 & $36.00-44.00$ & 230 \\
\hline $\mathrm{H}-12$ & 75 & 06 & 21 & $25.1^{\mathrm{C}}$ & & 14.11 & $29.00-42.73$ & 87 \\
\hline $\mathrm{P}, \mathrm{SC}$ & 75 & 07 & 20 & $25.1^{c}$ & & 13.90 & $29.00-43.00$ & 100 \\
\hline$H-13 A$ & 75 & 06 & 22 & & & 37.66 & $19.00-52.00$ & 710 \\
\hline St, Sc & 75 & $09^{\circ}$ & 30 & $17.3^{c}$ & & 37.63 & $20.00-52.00$ & 670 \\
\hline$H-13 B$ & 75 & 06 & 22 & & 26.70 & 38.01 & $33.00-41.44$ & 700 \\
\hline St, $C, g$ & 75 & 09 & 30 & $17.3^{\mathrm{C}}$ & 26.35 & 37.90 & $20.00-41.41$ & 720 \\
\hline$H-14 A$ & 75 & 06 & 22 & 31.4 & & $25.2^{\mathrm{e}}$ & $33.00-42.00$ & 260 \\
\hline $\mathrm{P}, \mathrm{Sc}$ & 75 & 10 & 01 & 31.9 & & $25.1^{\mathrm{e}}$ & $33.00-42.00$ & 260 \\
\hline
\end{tabular}


TABLE 2. Shallow temperatures and temperature gradients 16 for U.S. Geological Survey shallow test holes, Grass Valley, Nevada (continued)

\begin{tabular}{|c|c|c|c|c|c|c|c|c|}
\hline \multirow[b]{2}{*}{$\begin{array}{l}\text { Test } \\
\text { hole }\end{array}$} & \multicolumn{3}{|c|}{$\begin{array}{l}\text { Date of } \\
\text { measurcment }\end{array}$} & \multirow{2}{*}{$\begin{array}{l}\text { Depth to } \\
\text { saturated } \\
\text { zone } \\
(\mathrm{m})\end{array}$} & \multicolumn{2}{|c|}{$\begin{array}{l}\text { Temperature } \\
\left({ }^{\circ} \mathrm{C}\right)\end{array}$} & \multirow{2}{*}{$\begin{array}{l}\text { Depth interval } \\
\text { measured } \\
(\mathrm{m})\end{array}$} & \multirow{2}{*}{$\begin{array}{l}\text { Temperature } \\
\text { gradient } \\
\left({ }^{\circ} \mathrm{C} / \mathrm{km}\right)\end{array}$} \\
\hline & $\begin{array}{l}\frac{1}{0} \\
\stackrel{2}{2} \\
\stackrel{2}{>}\end{array}$ & $\stackrel{\circ}{E}$ & ते & & $15 \mathrm{~m}$ & $30 \mathrm{~m}$ & & \\
\hline$H-14 B$ & 75 & 06 & 22 & 31.4 & 20.35 & 25.04 & $10.00-18.00$ & 390 \\
\hline \multirow[t]{3}{*}{$P, C$} & & & & & & & $18.00-30.00$ & 300 \\
\hline & 75 & 09 & 30 & 31.9 & 20.11 & 25.01 & $10.00-16.00$ & 420 \\
\hline & & & & & & & $22.00-30.00$ & 300 \\
\hline$H-15$ & 75 & 06 & 21 & & 21.37 & 26.30 & $15.00-44.00$ & 300 \\
\hline \multirow[t]{4}{*}{$P, C$} & 75 & 07 & 20 & & 21.10 & 26.21 & $15.00-44.00$ & 310 \\
\hline & 75 & 09 & 07 & $33.8(?)^{\mathrm{N}}$ & 21.07 & 26.29 & $16.00-30.00$ & 340 \\
\hline & & & & & & & $30.00-44.39$ & 280 \\
\hline & 75 & 09 & 30 & $33.8(?)^{\mathrm{N}}$ & 20.93 & 26.25 & $34.00-44.00$ & 270 \\
\hline
\end{tabular}

$\mathrm{N}_{\text {determined from neutron logs. }}$

confined water level.

extrapolated linearly downward from lowest depth shown in depth interval, or upward from highest depth.

* Letters beneath hole number denote type of casing and completion:

$P$, plastic (PVC) casing.

St, steel casing.

C, casing capped at bottom.

Sc, screen at bottom of casing.

$\mathrm{g}$, indicates that the casing was grouted in (a1l other wells have the annulus backfilled with cuttings and/or dry surface materials). 
TABLE 3. Thermal conductivity and lithology of cores from test hole Q-1, Grass Valley, Nevada

\begin{tabular}{|c|c|c|c|}
\hline $\begin{array}{l}\text { Core depth } \\
\text { (meters) }\end{array}$ & Lithology & $\begin{array}{l}K \text { sample } \\
\text { depth } \\
\text { (meters) }\end{array}$ & $\left(\begin{array}{ccc}k \\
\mathrm{mcal} / \mathrm{cm} \mathrm{sec} & & \\
& & \mathrm{C}\end{array}\right)$ \\
\hline 62.48 to 64.01 & $\begin{array}{l}\text { Tan clay and silt; small peible sized } \\
\text { chert and quartzite clasts rare }\end{array}$ & $\begin{array}{l}62.73 \\
63.03 \\
63.31\end{array}$ & $\begin{array}{l}3.86 \\
4.09 \\
3.48\end{array}$ \\
\hline 121.92 to 123.44 & Tan clay and silt; $20 \%+$ medium sand & $\begin{array}{l}122.13 \\
122.22\end{array}$ & $\begin{array}{l}3.55 \\
3.84\end{array}$ \\
\hline 123.44 to 124.97 & $\begin{array}{l}\text { Tan clay and silt; } 2-5 \% \text { very coarse } \\
\text { sand; calcite cement }\end{array}$ & $\begin{array}{l}123.54 \\
123.75 \\
123.96 \\
124.15\end{array}$ & $\begin{array}{l}2.67 \\
3.38 \\
3.07 \\
3.38\end{array}$ \\
\hline 167.64 to 168.07 & Tan clay to very fine sand & $\begin{array}{l}167.79 \\
167.94 \\
168.01\end{array}$ & $\begin{array}{l}3.33 \\
3.26 \\
3.56\end{array}$ \\
\hline
\end{tabular}


TABLE 4. Thermal conductivity and lithology of cores from test hole Q-2, Grass Vaḷey, Nevada

Core depth (meters)
K sample depth $K$

Lithology

62.48 to 62.84 Conglomerate; quartzite, chert, basalt, 62.79 clast size 5-10 mm (maximum size $80 \mathrm{~mm}$ ), poor sorting, $20-30 \%$ tan clay and silt matrix

62.84 to 63.63 Conglomerate; average clast size 2-6

63.15

$\mathrm{mm}$ (maximum size $30 \mathrm{~mm}$ ), poor sorting, 63.43

$50-60 \%$ tan clay and silt matrix

122.53 to 123.09 .

Tan clay and silt; $5-20 \%$ poorly sorted clasts of quartzite, chert, and metavolcanics

123.09 to 124.05

Conglomerate; quartzite, chert, and metavolcanic clasts, $60-70 \%$ tan silt and clay matrix

124.05 to 124.46

Tan clay to very coarse sand; $10-20 \%$ chert clasts (maximum size $10 \mathrm{~mm}$ ), poor sorting

124.46 to 124.66

Conglomerate; average clast size 3-7 mm (maximum size $10 \mathrm{~mm}$ ), clasts predominantiy of chert, $50 \%+\tan$ clay to coarse sand matrix

124.66 to 125.22

Conglomerate; chert clasts predominate, average clast size $10-15 \mathrm{~mm}$ (maximum size $25 \mathrm{~cm}$ ), poor sorting, $50 \%+\tan$ clay to coarse sand matrix
122.80

123.08

123.20

3.54

124.36

124.66

3.85

124.97

125.21

3.64 
TABLE 5. Thermal conductivity and lithology of cores

from test hole Q-3, Grass Valley, Nevada

\begin{tabular}{|c|c|c|c|}
\hline $\begin{array}{l}\text { Core depth } \\
\text { (meters) }\end{array}$ & Lithology & $\begin{array}{l}K \text { sample } \\
\text { depth } \\
\text { (meters) }\end{array}$ & $\left.\frac{K}{(\text { mcal } / \mathrm{cm} \mathrm{sec}}{ }^{\circ} \mathrm{C}\right)$ \\
\hline 64.62 to 65.99 & $\begin{array}{l}\text { Conglomerate; clasts subrounded to } \\
\text { angular chert, average clast size } \\
5-15 \mathrm{~mm} \text { (maximum size } 50 \mathrm{~mm} \text { ), poor } \\
\text { sorting, } 40-60 \% \text { tan clay and silt } \\
\text { matrix }\end{array}$ & $\begin{array}{l}64.83 \\
65.07 \\
65.26 \\
65.59\end{array}$ & $\begin{array}{l}4.31 \\
5.02 \\
4.75 \\
4.95\end{array}$ \\
\hline 128.02 to $128.63^{\circ}$ & $\begin{array}{l}\text { Conglomerate; chert, quartzite and } \\
\text { rhyolitic clasts as large as } 30 \mathrm{~mm} \text {; } \\
50 \%+\text { tan clay to medium sand size } \\
\text { matrix }\end{array}$ & $\begin{array}{l}128.14 \\
128.47\end{array}$ & $\begin{array}{l}3.21 \\
5.17\end{array}$ \\
\hline 128.63 to 129.11 & Tan clay and silt; $5 \% \pm$ coarse sand & $\begin{array}{l}128.78 \\
129.05\end{array}$ & $\begin{array}{l}2.88 \\
3.24\end{array}$ \\
\hline 129.11 to 129.74 & Conglomerate & 129.45 & 6.05 \\
\hline 129.74 to 129.97 & Tan clay and silt & 129.88 & 3.50 \\
\hline 129.97 to 130.63 & Congl ome rate & 130.36 & 3.69 \\
\hline 164.59 to 165.91 & $\begin{array}{l}\text { Conglomerate; clasts of chert, } \\
\text { quartzite, and andesite, average } \\
\text { clast size } 1-5 \mathrm{~mm} \text { (maximum size } \\
70 \mathrm{~mm} \text { ), poor sorting }\end{array}$ & $\begin{array}{l}164.71 \\
165.02 \\
165.29 \\
165.63 \\
165.90\end{array}$ & $\begin{array}{l}4.42 \\
4.03 \\
4.81 \\
3.79 \\
3.73\end{array}$ \\
\hline
\end{tabular}


TABLE 6. Lithology and conductivity of cores from test we 11 QH-1, Grass Valley, Nevada

\begin{tabular}{|c|c|c|c|}
\hline $\begin{array}{l}\text { Core depth } \\
\text { (meters) }\end{array}$ & Lithology & $\begin{array}{l}\text { K sample } \\
\text { depth } \\
\text { (meters) }\end{array}$ & $\begin{array}{c}\mathrm{K} \\
\left(\mathrm{mcal} / \mathrm{cml} \sec { }^{\circ} \mathrm{C}\right)\end{array}$ \\
\hline 58.22 to 58.46 & $\begin{array}{l}\text { Conglomerate; clasts subrounded to } \\
\text { angular chert and quartzite, average } \\
\text { clast size } 10-30 \mathrm{~mm} \text {, poor sorting, } \\
30 \%+1 \text { ight green clay and silt } \\
\text { matrix }\end{array}$ & 58.37 & 5.45 \\
\hline 58.46 to 59.74 & $\begin{array}{l}\text { Light green silt and clay; perva- } \\
\text { sively sheared, moderately indurated, } \\
1-5 \% \text { sub-rounded to angular chert and } \\
\text { quartzite clasts }\end{array}$ & $\begin{array}{l}58.77 \\
59.10 \\
59.59\end{array}$ & $\begin{array}{l}4.18 \\
3.85 \\
3.20\end{array}$ \\
\hline 119.48 to 119.88 & $\begin{array}{l}\text { Fine sand; poor sorting, clayey } \\
\text { matrix }\end{array}$ & 119.60 & 3.97 \\
\hline 119.88 to 120.30 & $\begin{array}{l}\text { Medium to fine sand; poor sorting, } \\
\text { clayey matrix }\end{array}$ & & \\
\hline 120.30 to 121.31 & $\begin{array}{l}\text { Medium sand; poor sorting, clayey } \\
\text { matrix }\end{array}$ & $\begin{array}{l}120.43 \\
120.76\end{array}$ & $\begin{array}{l}3.78 \\
3.98\end{array}$ \\
\hline 152.70 to 152.89 & $\begin{array}{l}\text { Brown silt and clay; poor sorting, } \\
5-30 \% \text { very coarse sand }\end{array}$ & & \\
\hline 152.89 to 153.13 & $\begin{array}{l}\text { Brown silt and clay; } 5-20 \% \text { medium to } \\
\text { coarse pebble-sized chert and } \\
\text { quartzite clasts }\end{array}$ & 153.07 & 5.13 \\
\hline 153.13 to 153.22 & Brown silt and clay & 153.19 & 4.61 \\
\hline 153.22 to 154.23 & $\begin{array}{l}\text { Brown clay and coarse silt; thin in- } \\
\text { distinct interbeds with as much as } \\
10 \% \text { very coarse sand }\end{array}$ & $\begin{array}{l}153.38 \\
153.56 \\
154.23\end{array}$ & $\begin{array}{l}4.40 \\
2.98 \\
4.13^{\star}\end{array}$ \\
\hline
\end{tabular}

*density: 2.32 ; porosity: $16.6 \%$ 
TABLE 7. Lithology and conductivity of cores from test we $11^{\circ} \mathrm{QH}-2$, Grass Valley, Nevada

\begin{tabular}{|c|c|c|c|}
\hline $\begin{array}{l}\text { Core depth } \\
\text { (meters) }\end{array}$ & Lithology & $\begin{array}{l}\text { K sample } \\
\text { depth } \\
\text { (meters) }\end{array}$ & $\begin{array}{c}\mathrm{K} \\
\left(\mathrm{mcal} / \mathrm{cm} \sec { }^{\circ} \mathrm{C}\right)\end{array}$ \\
\hline 60.96 to 62.79 & $\begin{array}{l}\text { Light green clay and silt; thin in- } \\
\text { distinct coarse silt to very fine } \\
\text { sand interbeds, evidence of minor } \\
\text { shearing, trace of secondary pyrite } \\
\text { along fractures }\end{array}$ & $\begin{array}{l}61.60 \\
61.78 \\
61.94 \\
61.96 \\
62.06 \\
62.36 \\
62.51 \\
62.73\end{array}$ & $\begin{array}{l}2.55 \\
2.61 \\
2.79 \\
2.75 \\
2.91 \\
2.77 \\
2.79 \\
2.79\end{array}$ \\
\hline 121.92 to 122.41 & $\begin{array}{l}\text { Light green clay to coarse silt; } \\
1-2 \% \text { chert clasts, average clast } \\
\text { size } 2-6 \mathrm{~mm}\end{array}$ & 122.16 & 2.61 \\
\hline 122.41 to 124.00 & Light green clay to medium silt & $\begin{array}{l}122.53 \\
122.70 \\
122.90\end{array}$ & $\begin{array}{l}2.90 \\
3.06 \\
2.46\end{array}$ \\
\hline 152.70 to 154.53 & $\begin{array}{l}\text { Light green silt to very fine sand; } \\
\text { evidence of minor shearing, trace } \\
\text { of secondary calcite as veining }\end{array}$ & $\begin{array}{l}153.04 \\
153.22 \\
153.86 \\
154.14 \\
154.53\end{array}$ & $\begin{array}{l}3.32 \\
2.92 \\
3.26 \\
3.48 \\
3.57^{\star}\end{array}$ \\
\hline
\end{tabular}

*density: 2.04 ; porosity: $27.1 \%$ 
TABLE 8. Lithology and conductivity of cores from test well QH-3, Grass Valley, Nevada

Core depth

(meters)
Lithology
K sample

depth

(meters) (mcal/cm sec $\left.{ }^{\circ} \mathrm{C}\right)$
60.96 to 61.87 Conglomerate; clasts subrounded to angular quartzite, average clast size 15-25 mm (maximum size $65 \mathrm{~mm}$ ), poor sorting, $20 \% \pm \tan$ silt and clay matrix
61.11

61.17

4.98

5.33

119.02

119.18

119.36

119.48

3.81

5.59

3.11

4.32

152.70 to 152.86 Tan clay and silt; <10\% pebble-sized clasts

152.86 to 153.01

Conglomerate; average clast size $3-8 \mathrm{~mm}$ (maximum size $40 \mathrm{~mm}$ ), $20 \% \pm$ tan clay and silt matrix.

152.89

4.70

152.98

4.90

153.19

3.60

153.01 to $153.40 \quad$ Conglomerate; average clast size
$20-40 \mathrm{~mm}$ (maximum $85 \mathrm{~mm}$ ), predominantly chert, quartzite, and metavolcanic clasts, with some schistose and granitic fragments, $20 \% \pm$ clay and silt matrix 
TABLE 9. Lithology and conductivity of cores from test we $11 \mathrm{QH}-4$, Grass Valley, Nevada

\begin{tabular}{|c|c|c|c|}
\hline $\begin{array}{l}\text { Core depth } \\
\text { (meters) }\end{array}$ & Lithology & $\begin{array}{l}\text { K sample } \\
\text { depth } \\
\text { (meters) }\end{array}$ & $\begin{array}{c}\mathrm{k} \\
\left.(\mathrm{mca}) / \mathrm{cm} \sec { }^{\circ} \mathrm{C}\right)\end{array}$ \\
\hline 58.22 to 58.83 & $\begin{array}{l}\text { Conglomerate; chert and quartzite } \\
\text { clasts predominate, } 30 \%+\tan \text { clay } \\
\text { and silt matrix }\end{array}$ & $\begin{array}{l}58.43 \\
58.49\end{array}$ & $\begin{array}{l}4.01 \\
4.06\end{array}$ \\
\hline 58.83 to 59.15 & $\begin{array}{l}\text { Conglomerate; clasts subrounded to } \\
\text { angular, } 75 \% \text { of clasts are chert and } \\
\text { quartzite, remainder are metavolcanic } \\
\text { and andesitic, } 20 \% \pm \text { tan clay and } \\
\text { silt matrix }\end{array}$ & 59.04 & 4.22 \\
\hline 59.15 to 59.45 & $\begin{array}{l}\text { Tan clay and silt; } 2-5 \% \text { very coarse, } \\
\text { sand }\end{array}$ & 59.22 & 2.84 \\
\hline 59.45 to 59.85 & $\begin{array}{l}\text { Tan clay and silt; } 20-40 \% \text { clasts as } \\
\text { large as } 20 \mathrm{~mm}\end{array}$ & $\begin{array}{l}59.50 \\
59.59\end{array}$ & $\begin{array}{l}3.04 \\
3.04\end{array}$ \\
\hline 122.00 to 123.00 & $\begin{array}{l}\text { Conglomerate; quartzite, chert and } \\
\text { graywacke clasts as large as } 100 \mathrm{~mm} \text {, } \\
10 \%+\text { brick-red clay matrix }\end{array}$ & 122.59 & 4.19 \\
\hline 154.53 to 156.06 & $\begin{array}{l}\text { Brick-red clay; high angle }\left(80^{\circ}\right) \\
\text { shear planes }\end{array}$ & $\begin{array}{l}154.69 \\
154.96 \\
155.51 \\
155.97\end{array}$ & $\begin{array}{l}2.68 \\
2.94 \\
2.80 \\
3.01\end{array}$ \\
\hline
\end{tabular}


TABLE 10. Generalized lithology of Grass Valley test holes (based on examination of drill cuttings)

Silt, clayey


TABLE 1C. continued)

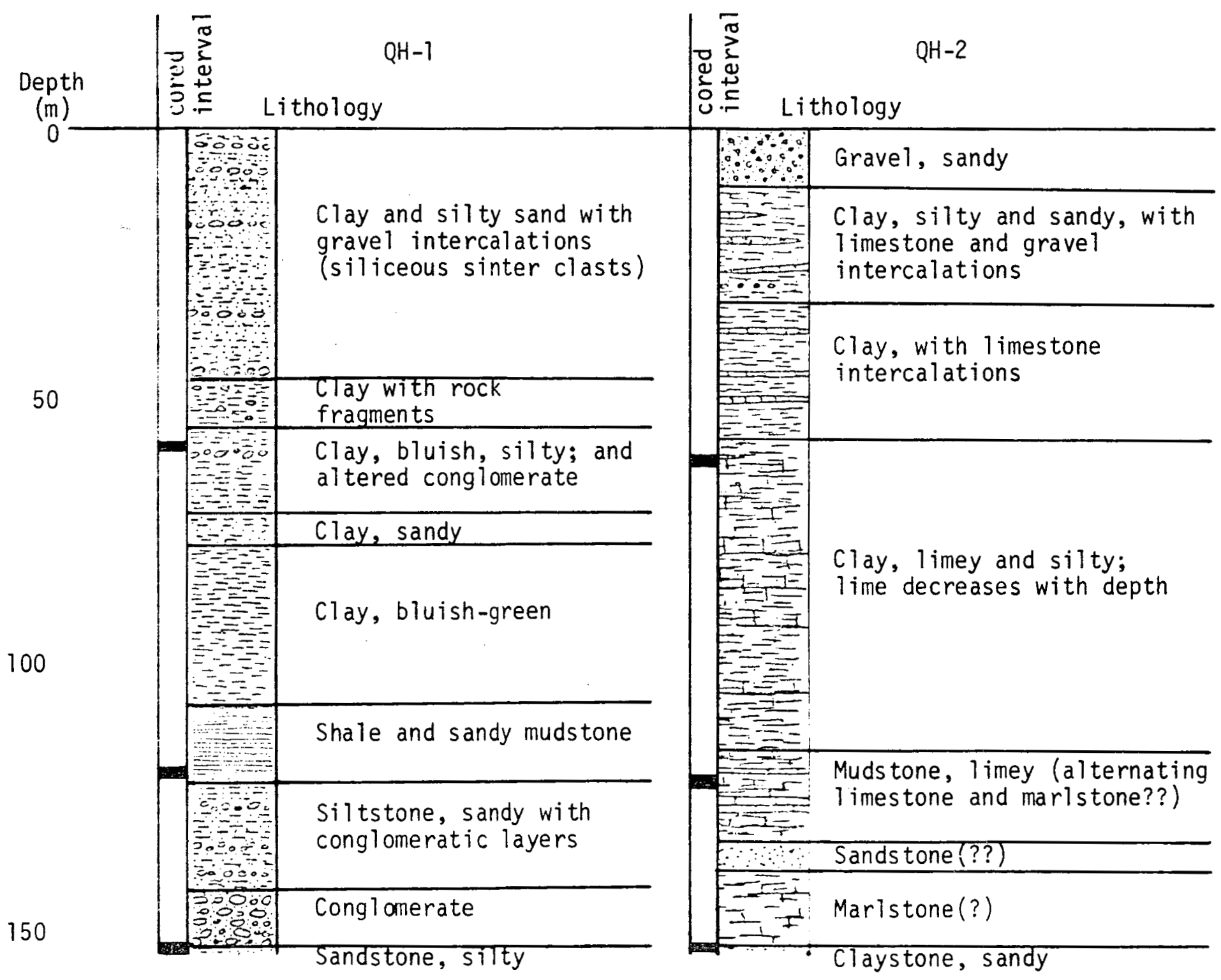


TABLE 10. =ontinued)

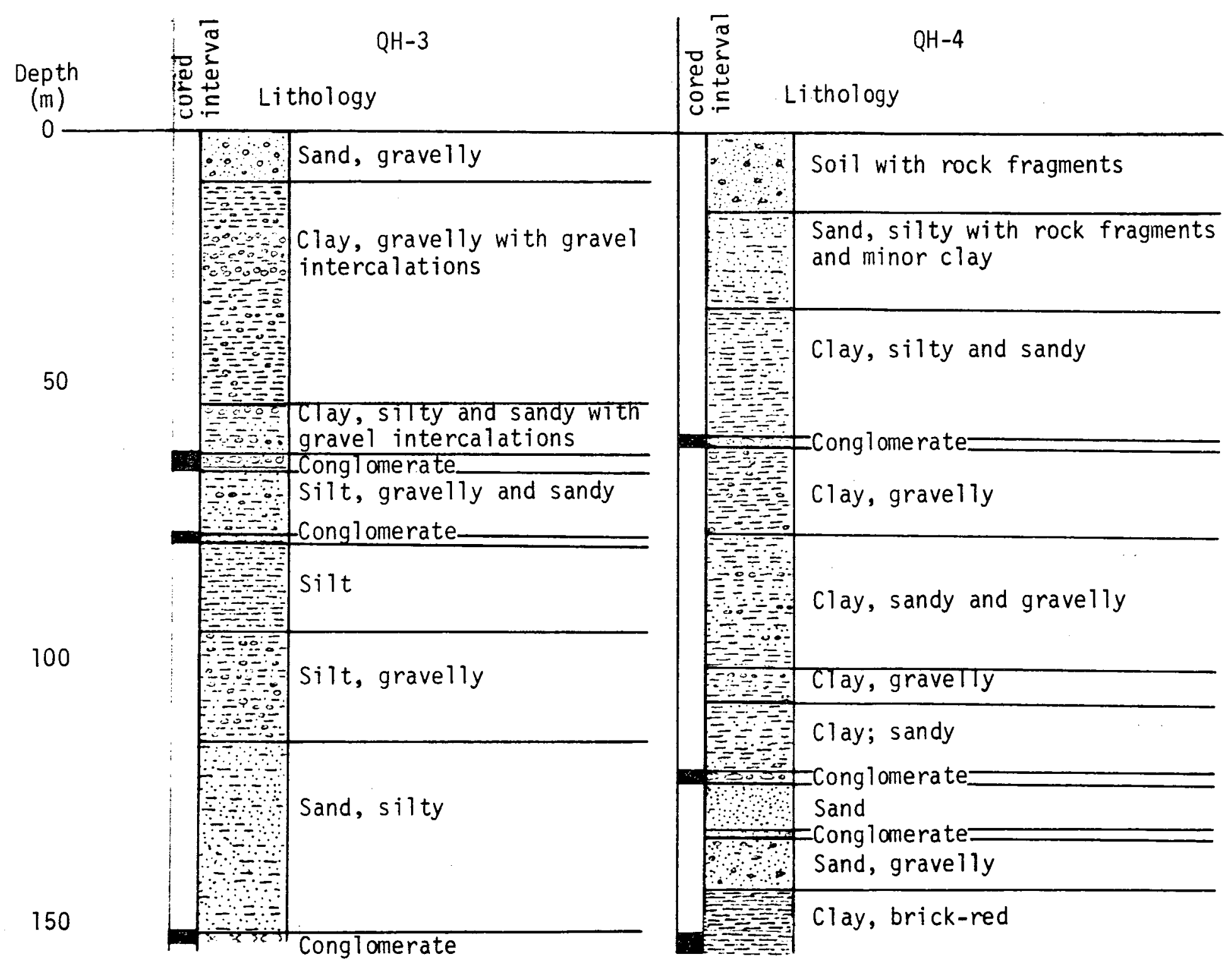


TABLE 11. Summary of temperature gradients, conductivities, and heat flows for holes near Buffalo Valley Hot Springs, Nevada

\begin{tabular}{|c|c|c|c|c|}
\hline Hole & Interval & $\begin{array}{l}\text { Grad } \\
{ }^{\circ} \mathrm{C} / \mathrm{km}\end{array}$ & $\begin{array}{c}\mathrm{K} \\
\mathrm{mcal} / \mathrm{cm} \text { sec }{ }^{\circ} \mathrm{C}\end{array}$ & $\stackrel{q}{\mathrm{HFU}}$ \\
\hline \multirow[t]{3}{*}{1} & $30-37$ & 147 & $3.7^{\star}$ & 5.5 \\
\hline & $45-60$ & $280^{\dagger}$ & 2.65 & 7.4 \\
\hline & & & average & $6.5+1$ \\
\hline 2 & $45-116$ & 79 & 2.48 & 2.0 \\
\hline 3 & $40-97$ & 107.5 & 2.57 & 2.8 \\
\hline
\end{tabular}

*Mean of the two extreme outcrop specimen values for basalt (3.08 and 4.36$)$.

${ }^{\dagger}$ Based on a temperature $\log$ made only a few hours after cessation of drilling. 
TABLE 12. Lithology and thermal conductivity for conductivity samples from Buffalo Valley heat-flow holes

\begin{tabular}{|c|c|c|c|}
\hline Hole \# & Depth & Li thol ogy* & $\stackrel{\mathrm{Kcal} / \mathrm{cm} \mathrm{sec}{ }^{\circ} \mathrm{C}}{ }$ \\
\hline \multirow[t]{4}{*}{1} & $0^{+}$ & $\begin{array}{c}\text { basalt } \\
p=2.12, \phi=20.6\end{array}$ & 3.08 \\
\hline & $0^{+}$ & $p=2.69, \phi=7.8$ & 4.10 \\
\hline & $0^{\dagger}$ & $\rho=2.71, \phi=7.0$ & 4.36 \\
\hline & 67.7 & silty sand & 2.65 \\
\hline \multirow[t]{5}{*}{2} & 92.6 & clayey sand & 2.36 \\
\hline & $93.0\}$ & & 2.36 \\
\hline & 113.71 & & 2.52 \\
\hline & 114.0 & gritty sand & 2.66 \\
\hline & 114.3 & & 2.54 \\
\hline \multirow[t]{8}{*}{3} & $61.6)$ & & 2.62 \\
\hline & 61.9 & silty sand & 2.42 \\
\hline & 62.2 & & 2.34 \\
\hline & 62.5 & & 2.36 \\
\hline & 78.3 & silty sand & 2.39 \\
\hline & 95.11 & & 2.70 \\
\hline & $96.0\}$ & silty clay & 2.59 \\
\hline & $96.6\}$ & & 3.38 \\
\hline
\end{tabular}

*For basalts $\rho=$ density $\mathrm{g} / \mathrm{cm}^{3}, \phi=$ apparent porosity $\%$. Nearby outcrop samples of basalt flow intersected between 1,30 and 1,37 meters. 


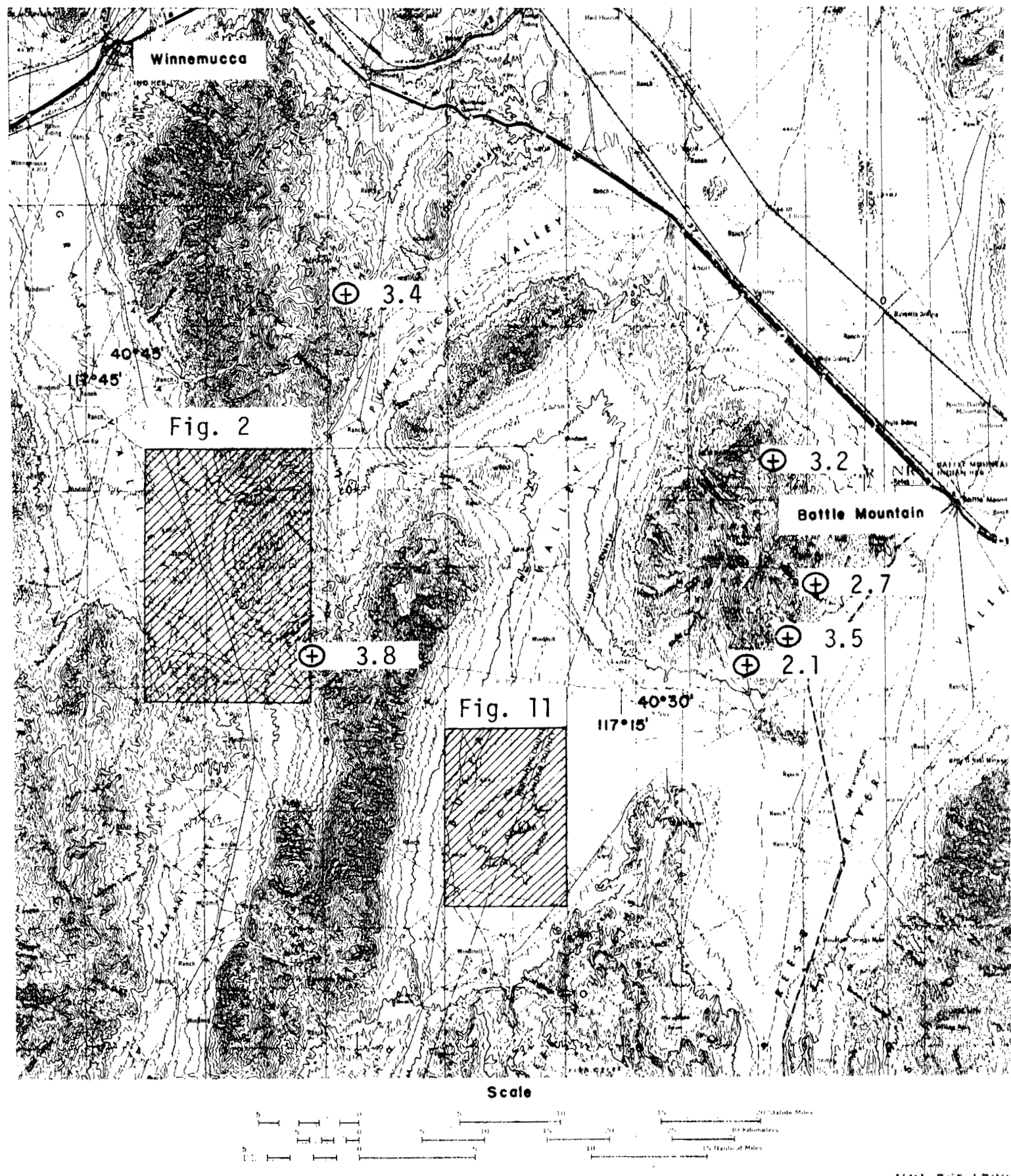

XiB!, 763.1720

Figure 1. Location map showing areas of more detailed maps together with previously published heat-flow values $\oplus$. 

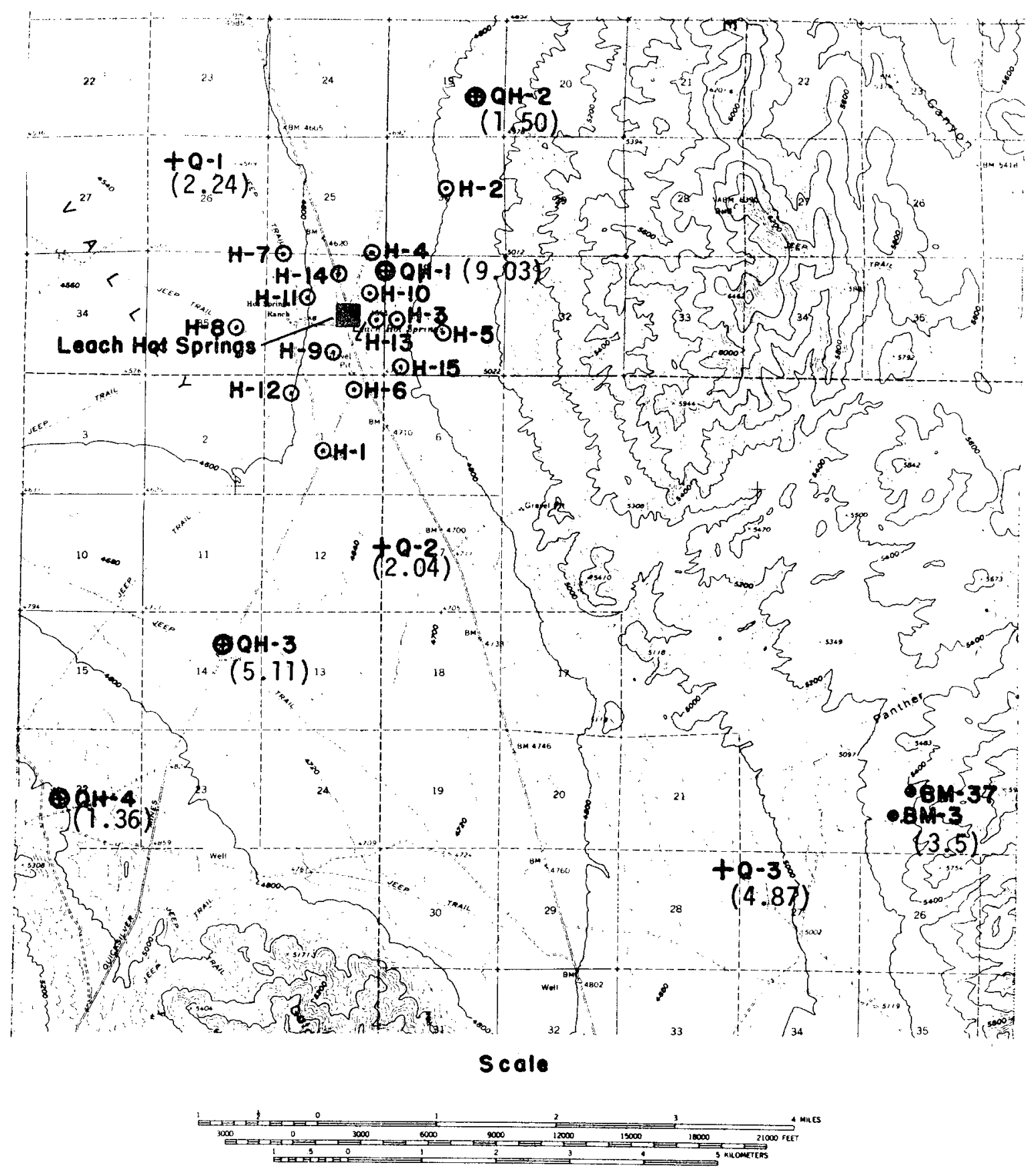

Figure 2. Locations of wells at Grass Valley (heat-flow

XBL $765-1719$ values are shown in parentheses).

+ Q, heat-flow holes

$\odot H$, shallow hydrologic test wells

$\oplus \mathrm{QH}$, heat flow-hydrologic test wells

heat-flow determinations from Sass and others, 1971 


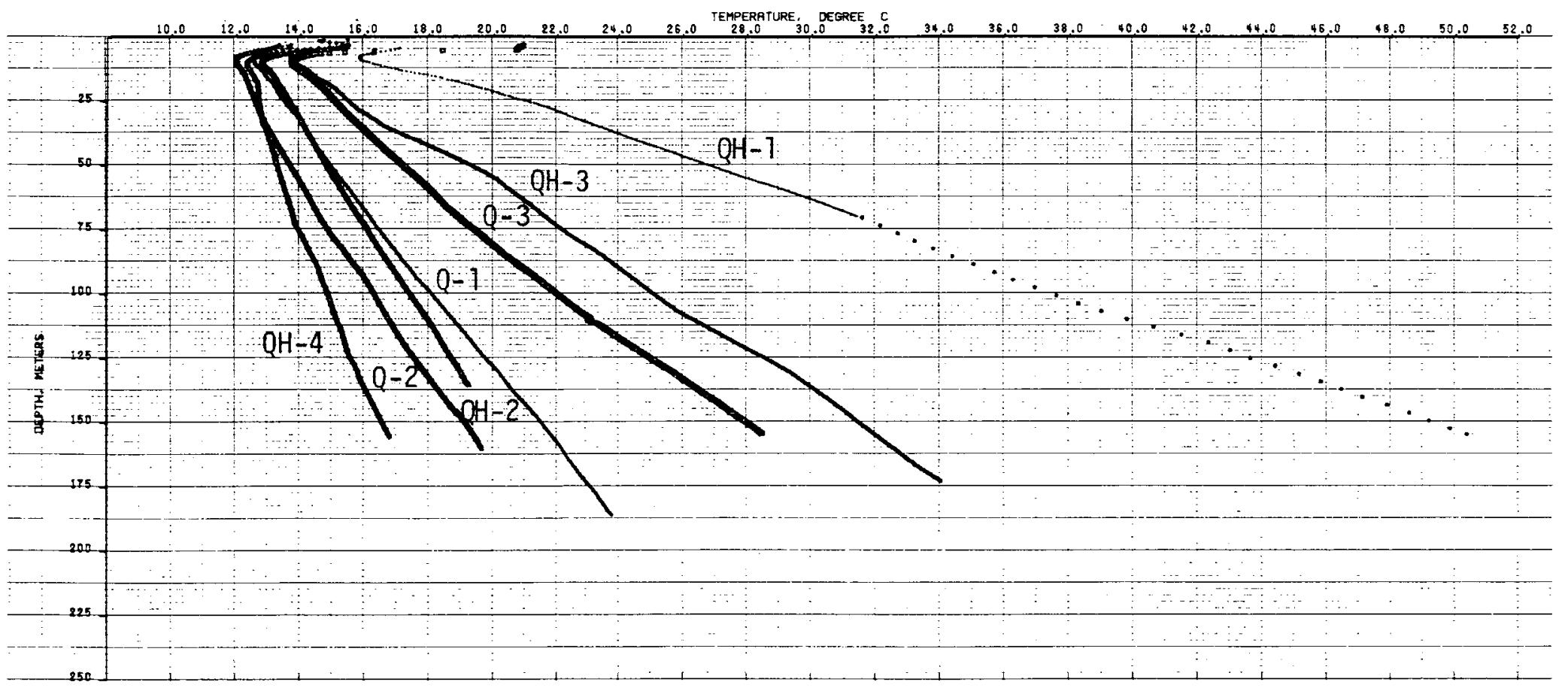

$X B 1.25 \div 2$

Figure 3. Temperature profiles for holes $Q-1$ through $Q-3$ and $Q H-1$ through $Q H-4$, Grass Valley, Nevada. All temperatures measured on 10-21-75. 


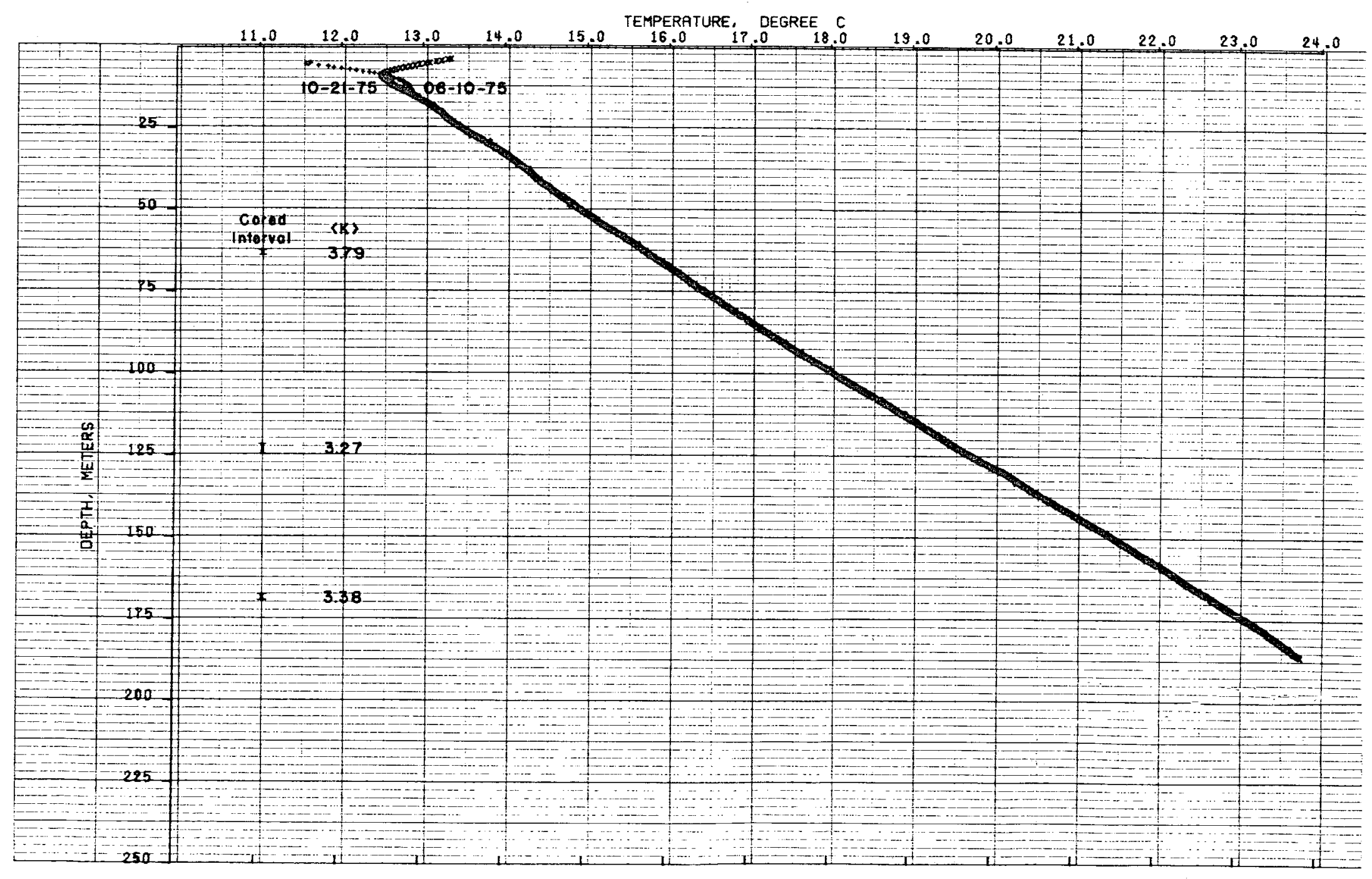

Figure 4. Temperature profiles from hole Q-1, Grass Valley, drilled 4-5 to $4-8,1975$. 


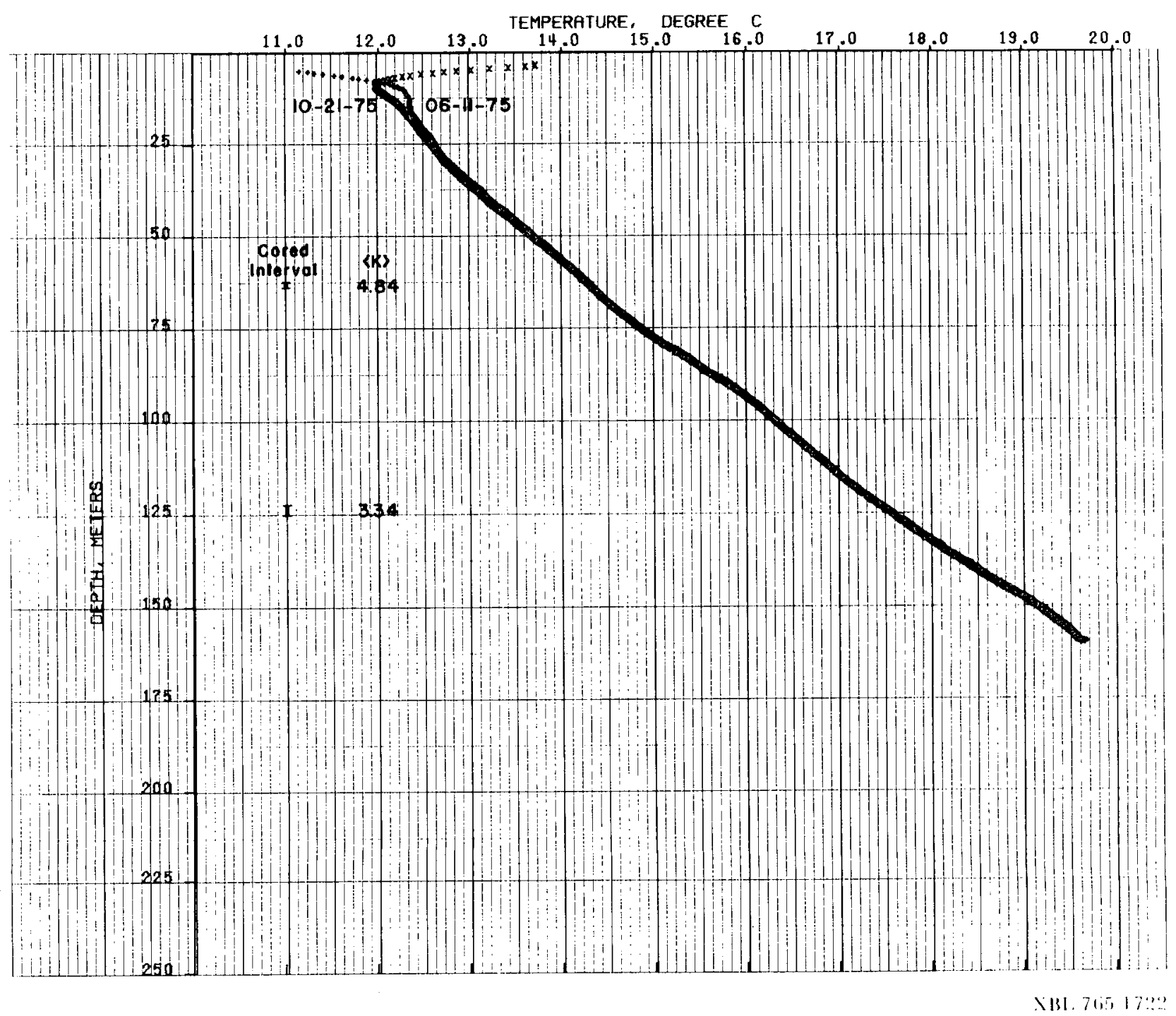

Figure 5. Temperature profiles from hole Q-2, Grass Valley, drilled 4-9 to 4-17, 1975 (includes 4-day break). 


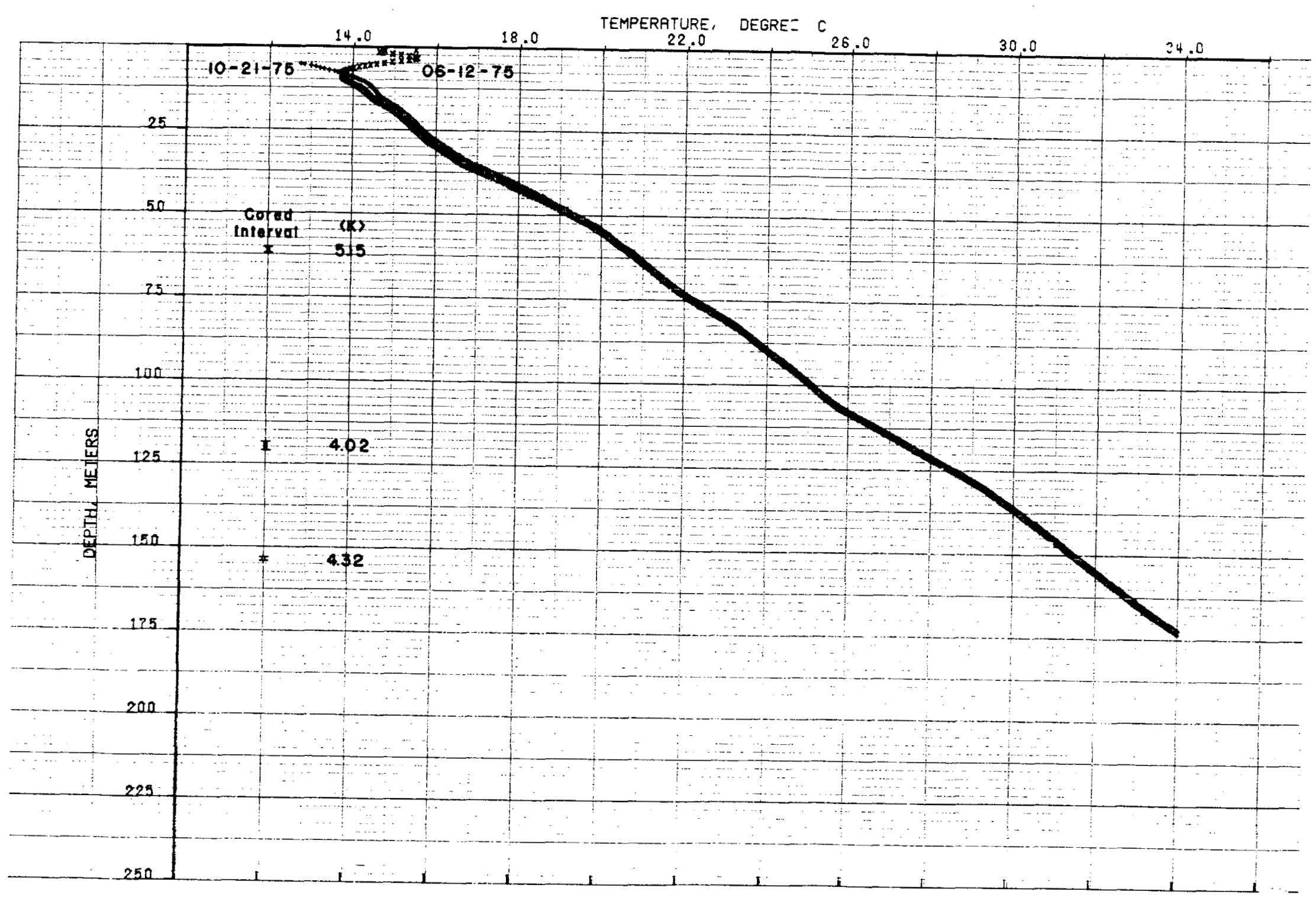

Figure 6. Temperature profiles from hole Q-3, Grass Valley, drilled 4-18

XBI. $755-1726$ to $4-22,1975$. 


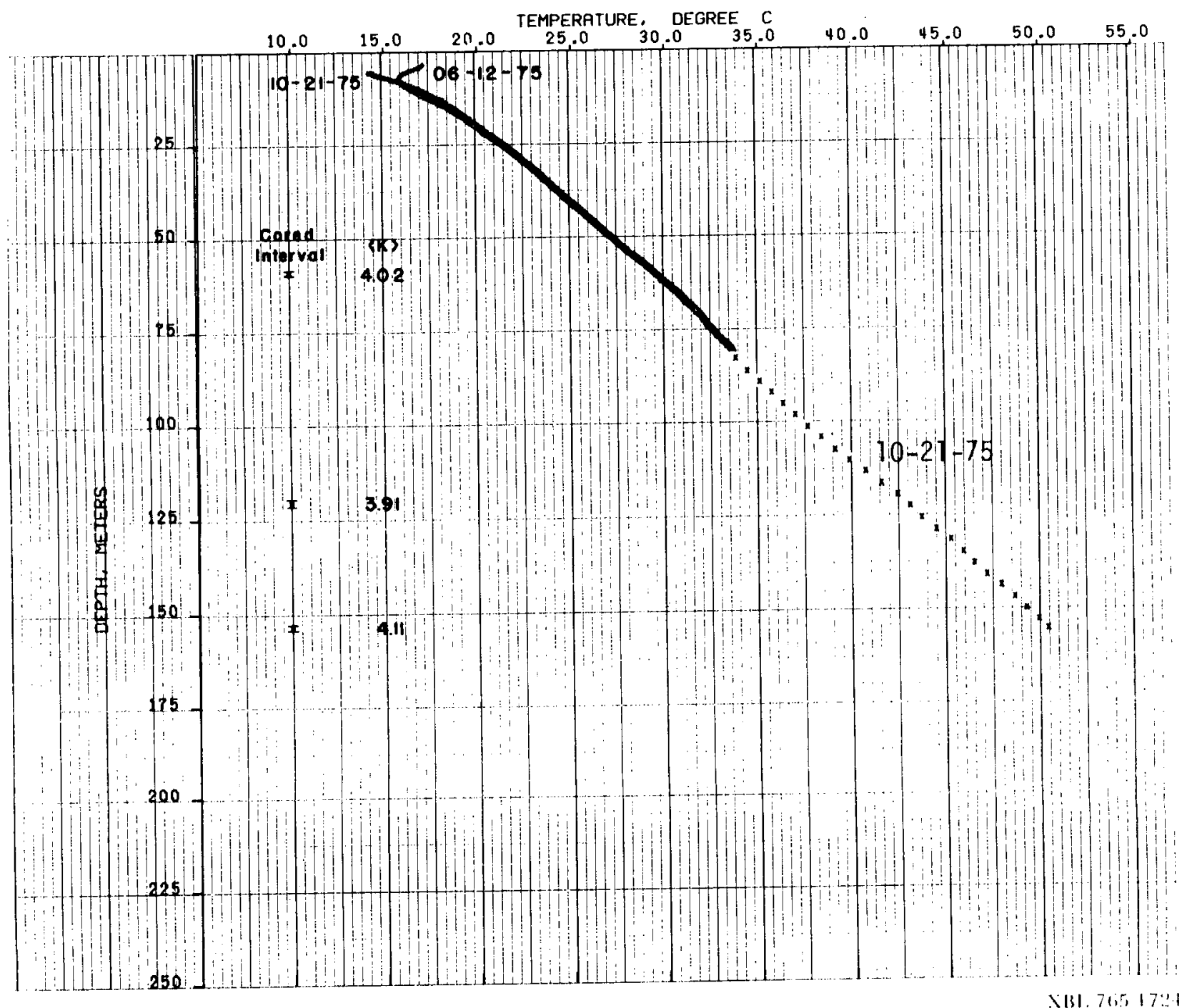

Figure 7. Temperatures from hole QH-1, Grass Valley, drilled 4-23 to 5-3, 1975 (includes 4-day break). 


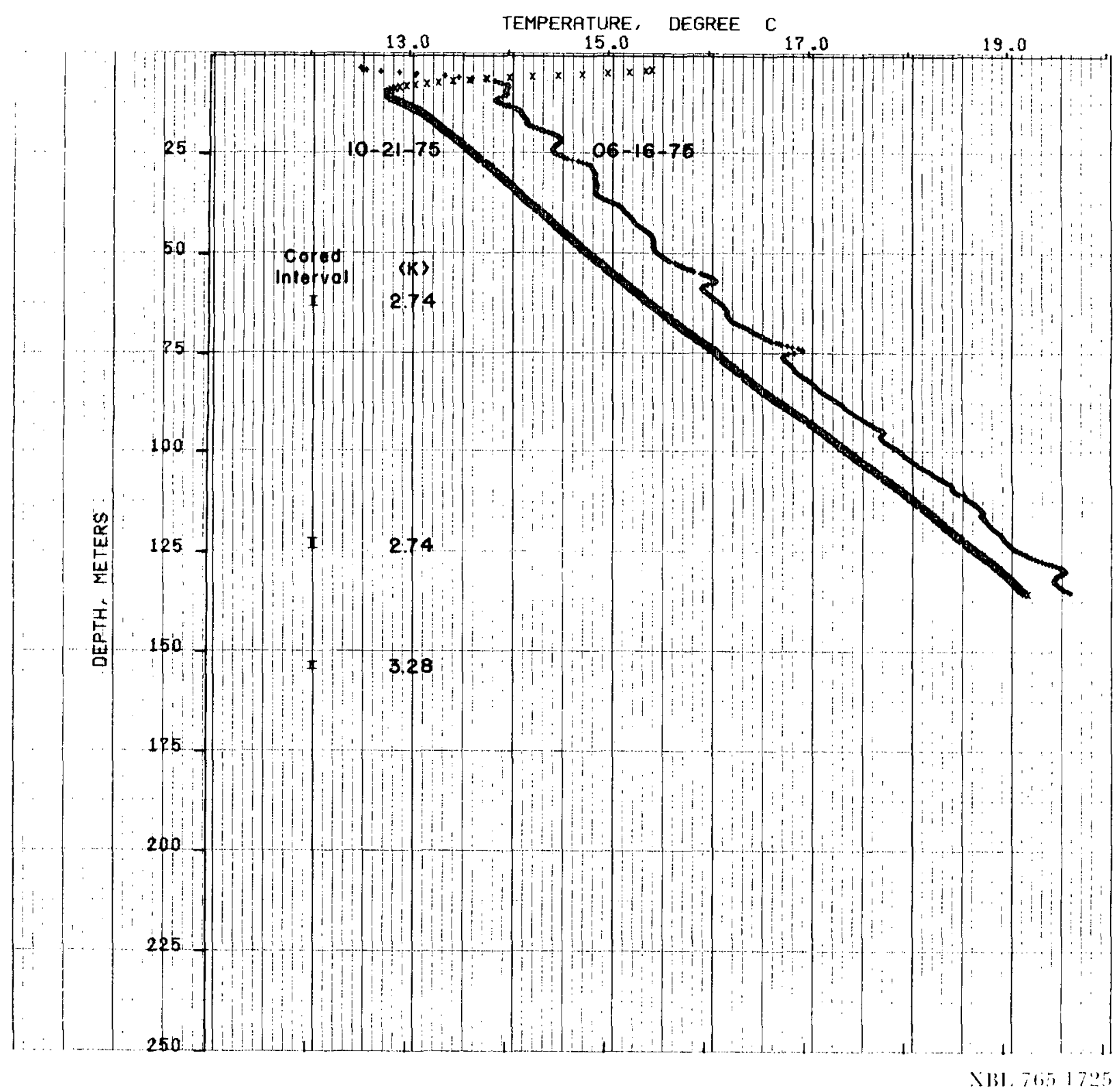

Figure 8. Temperatures from hole $\mathrm{QH}-2$, Grass Valley, drilled $6-1$ to $6-4,1975$. 


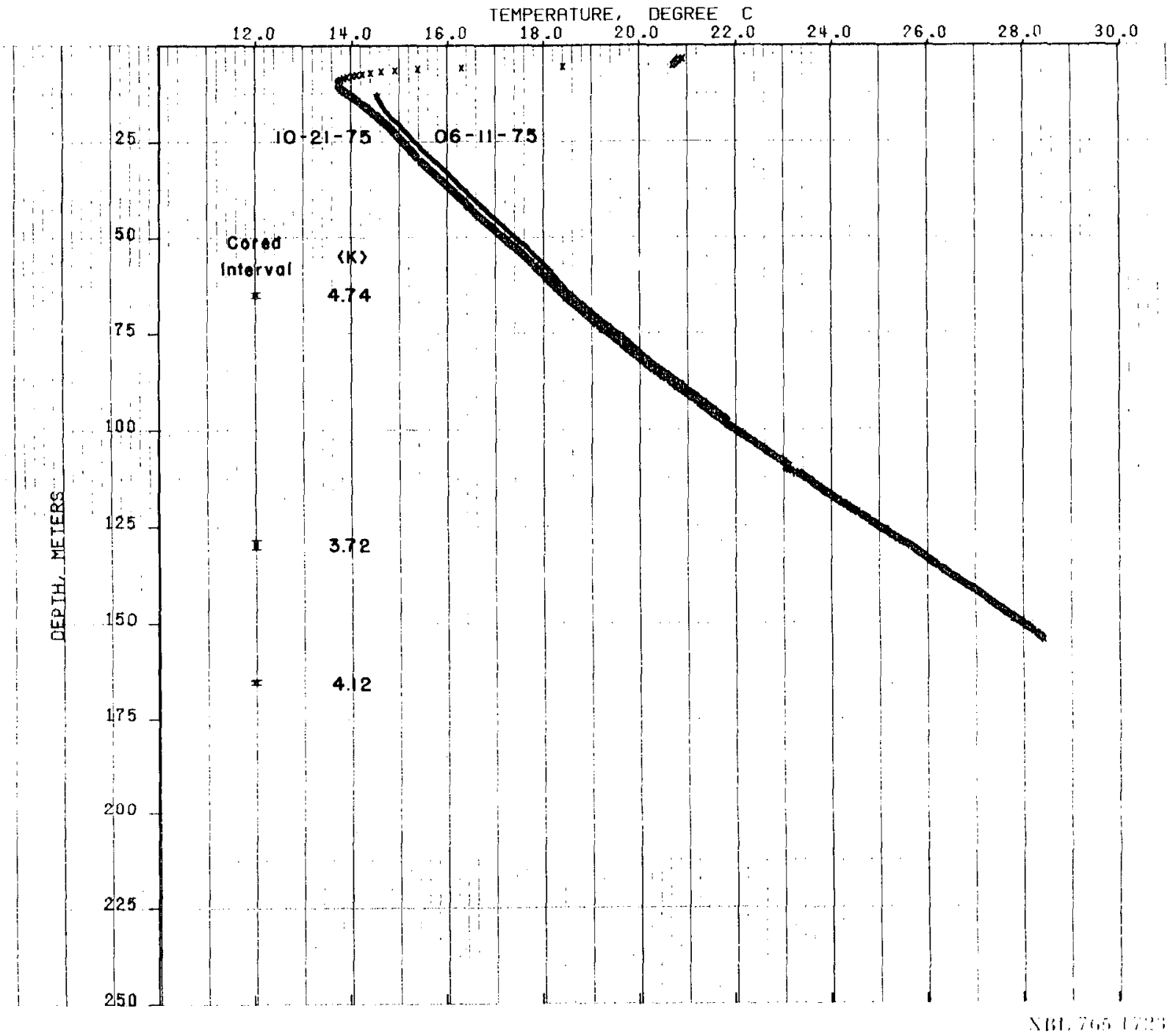

Figure 9. Temperatures from hole $\mathrm{QH}-3$, Grass Valley, drilled $5-5$ to $5-16,1975$ (includes 4-day break). 


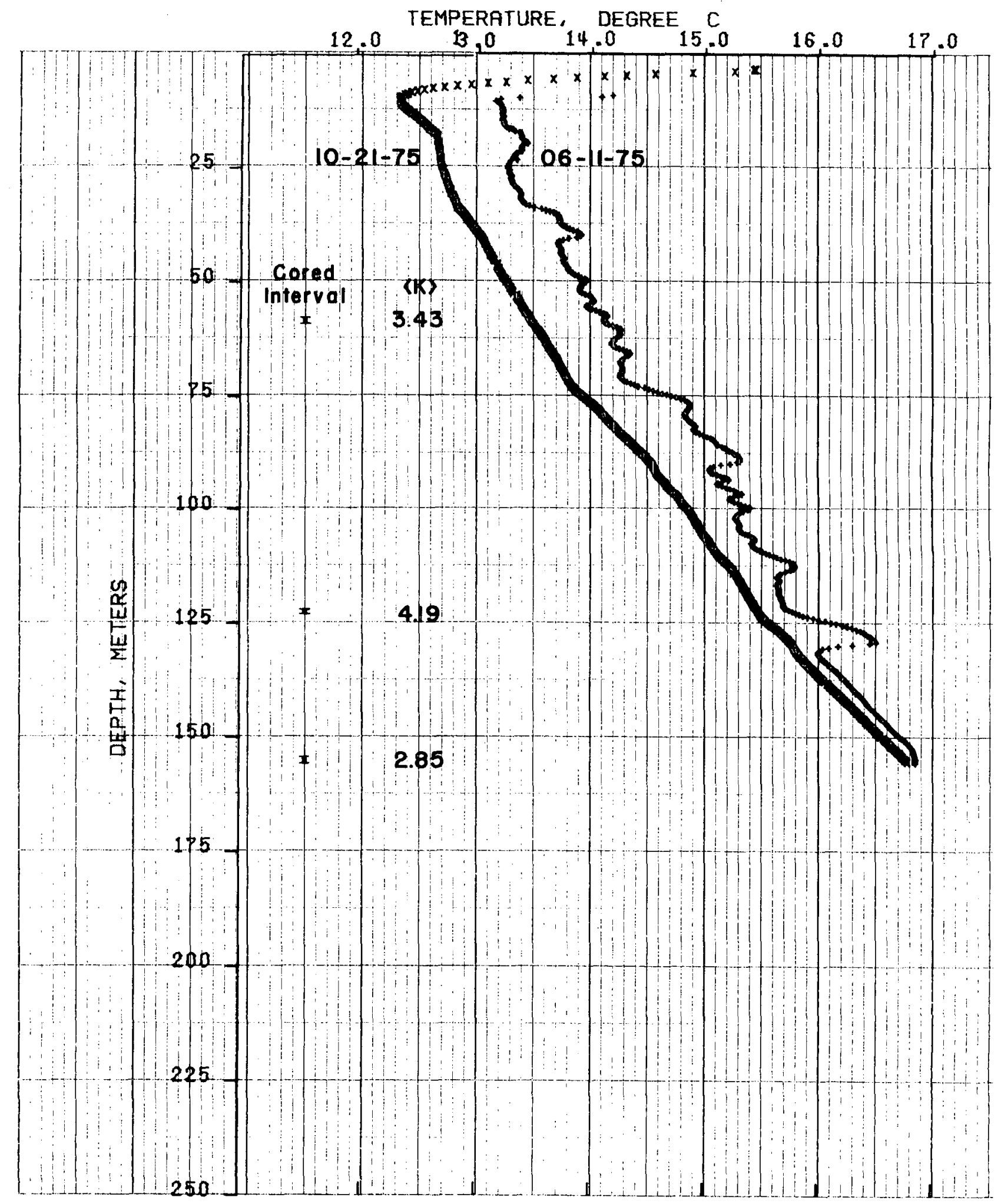

Figure 10. Temperatures from hole $\mathrm{QH}-4$, Grass Valley, drilled 5-20 to 5-30, 1975 (includes 5-day break). 

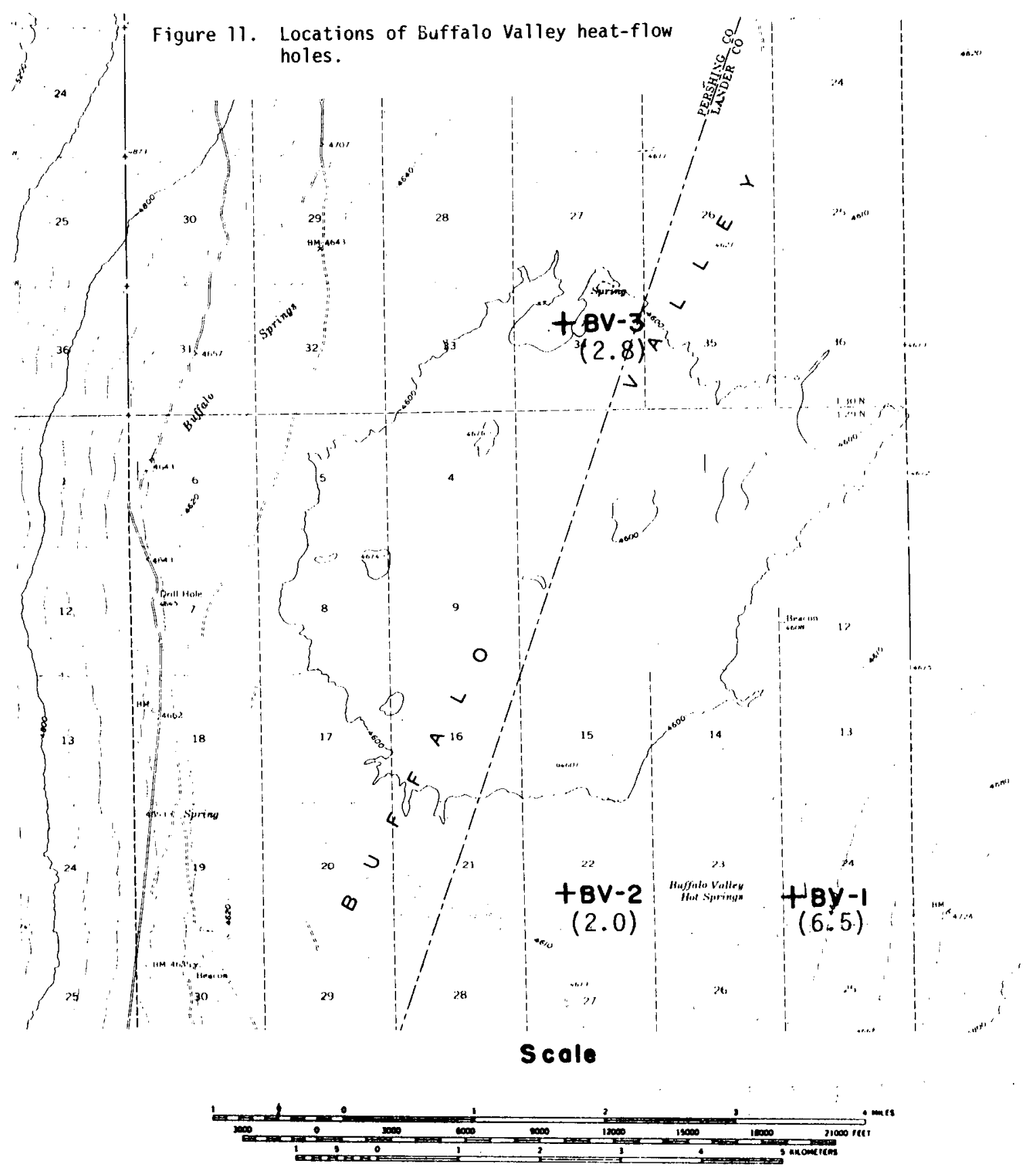

xB1. 765.1718 


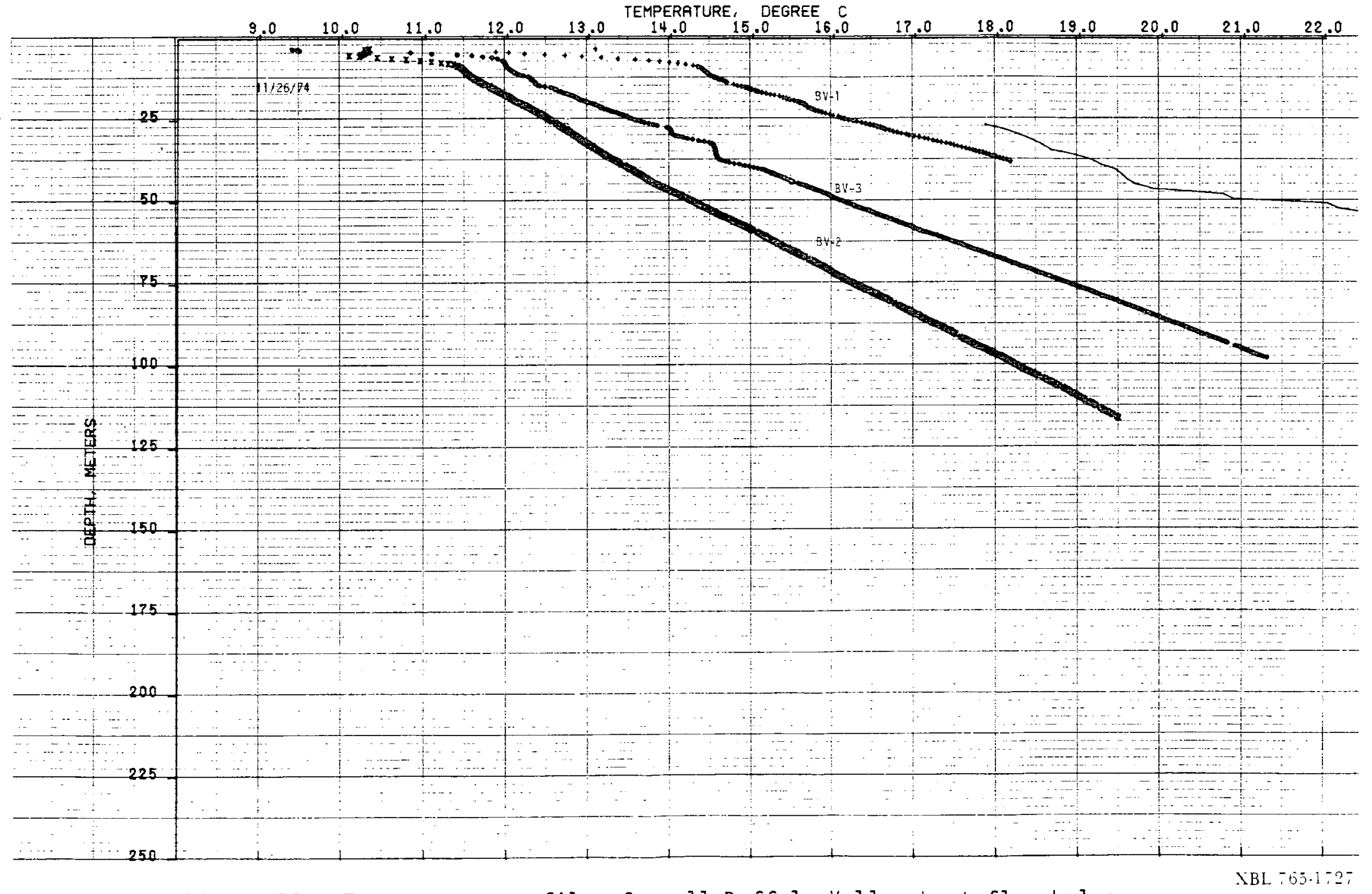

Figure 12. Temperature profiles for all Buffalo Valley heat-flow holes. 


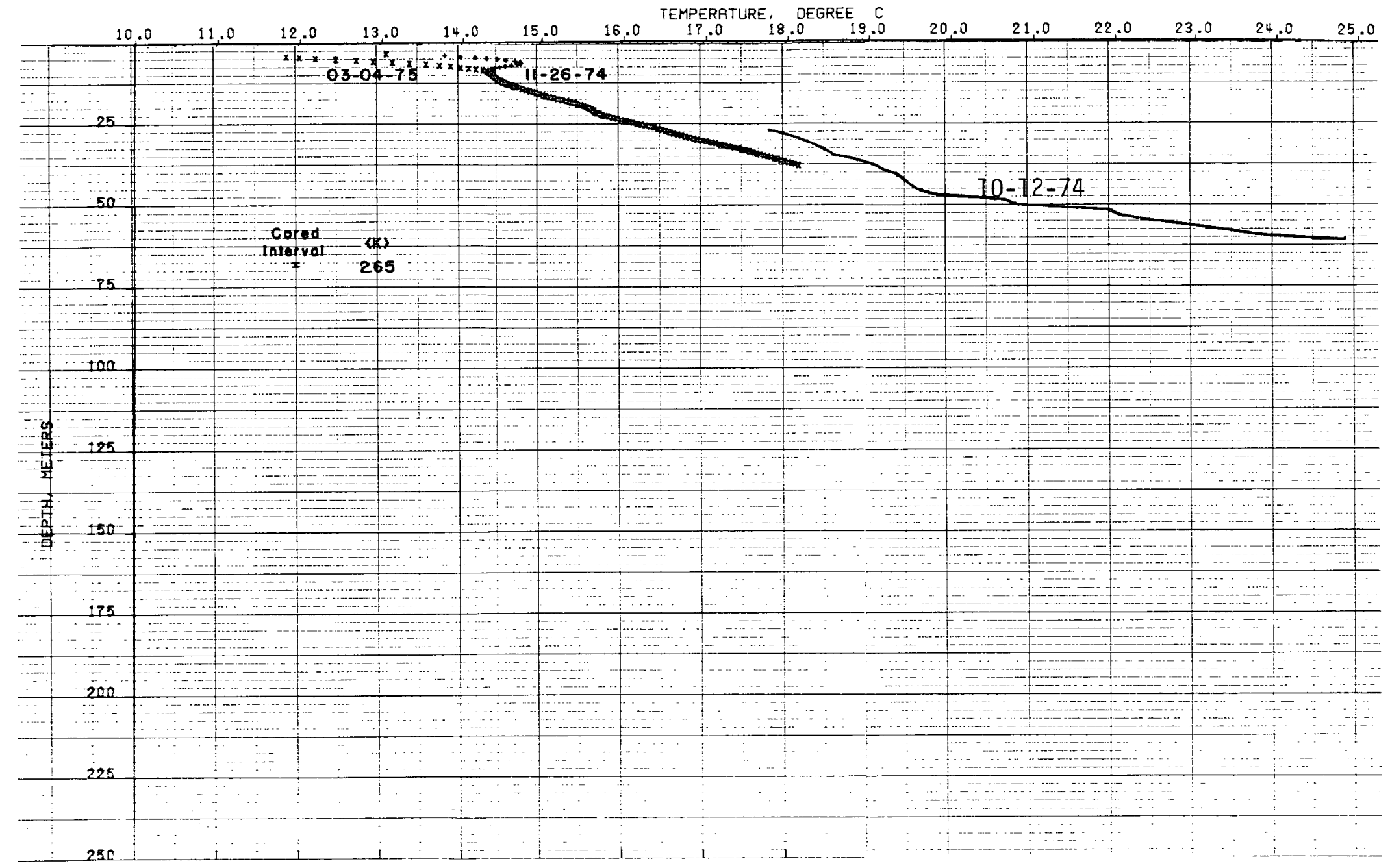

Figure 13. Temperature profiles for heat-flow hole BV-1, Buffalo Valley, drilled 10-9 XBL.-65-1.30 to $10-13,1974$. 
Figure 14. Temperature profiles for heat-flow hole BV-2, Buffalo Valley, drilled 10-14 to 10-16, 1974.

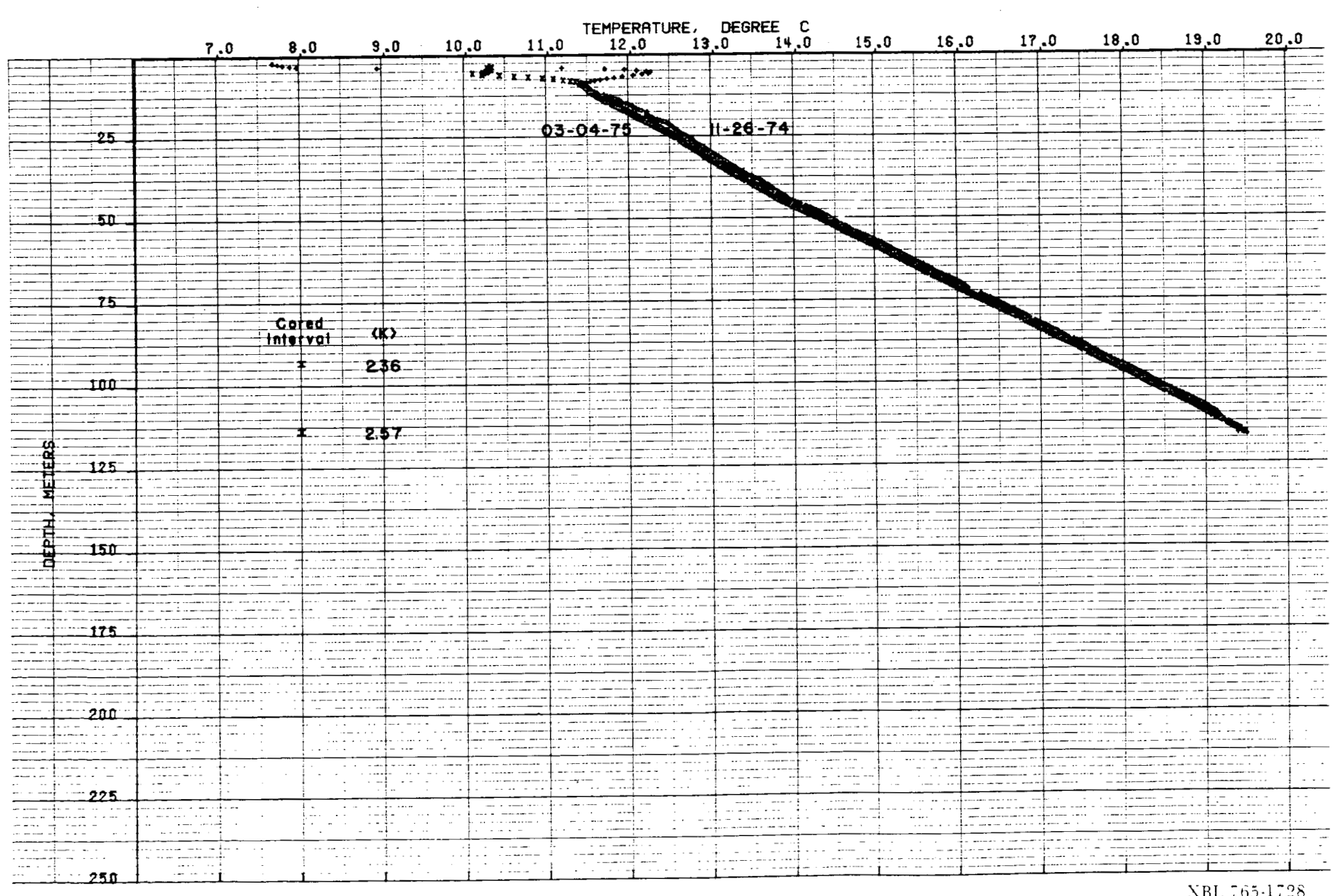


Figure 15. Temperature profiles for heat-flow hole BV-3, Buffalo Valley, drilled 10-17 to 10-18, 1974.

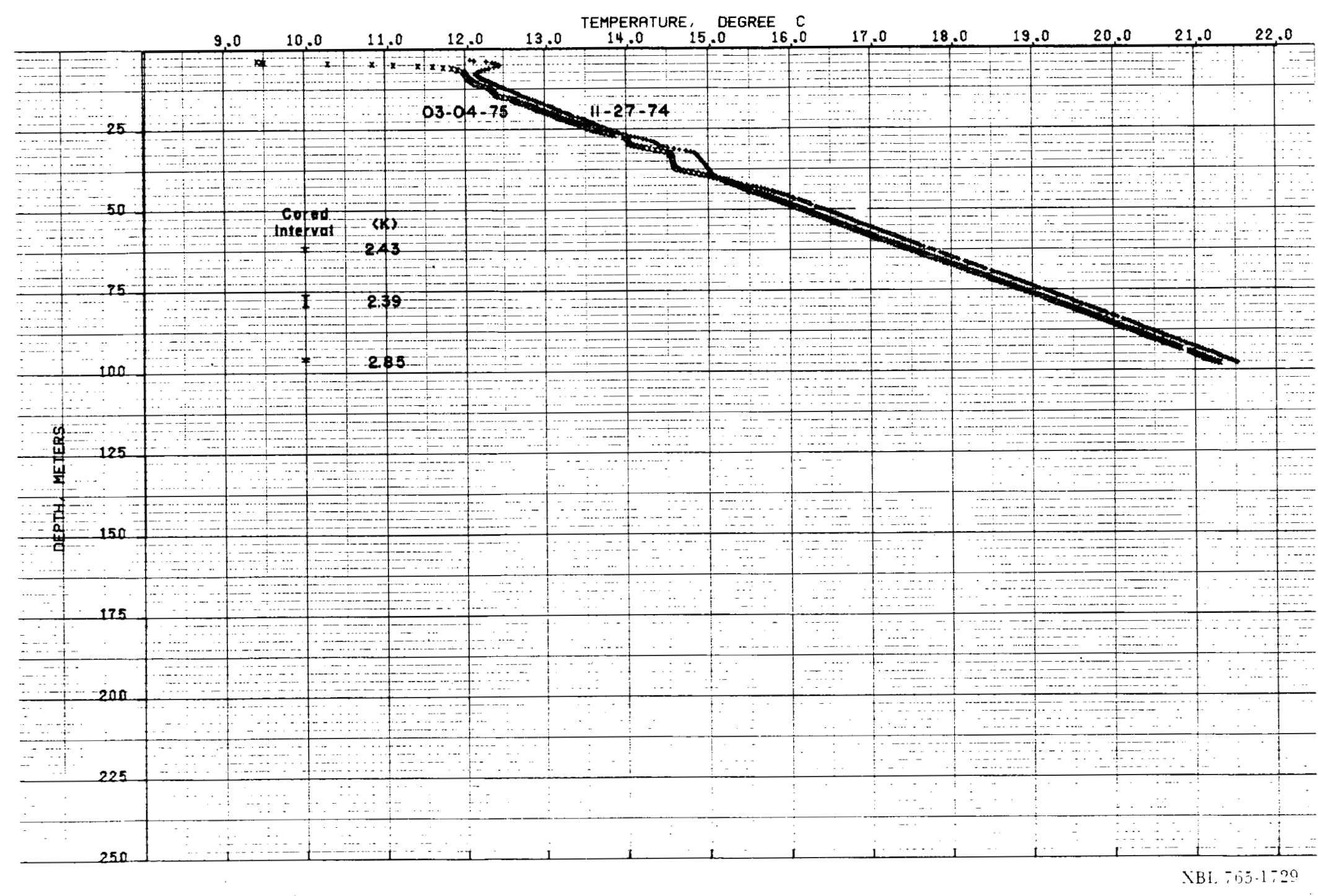

